ALEA, Lat. Am. J. Probab. Math. Stat. 16, 729-757 (2019)

DOI: $10.30757 /$ ALEA.v16-26

\title{
Functional convergence for moving averages with heavy tails and random coefficients
}

\section{Danijel Krizmanić}

Department of Mathematics, University of Rijeka

Radmile Matejčić 2, 51000 Rijeka, Croatia

E-mail address: dkrizmanic@math.uniri.hr

URL: http://www.math.uniri.hr/ dkrizmanic/

Abstract. We study functional convergence of sums of moving averages with random coefficients and heavy-tailed innovations. Under some standard moment conditions and the assumption that all partial sums of the series of coefficients are a.s. bounded between zero and the sum of the series we obtain functional convergence of the corresponding partial sum stochastic process in the space $D[0,1]$ of càdlàg functions with the Skorohod $M_{2}$ topology.

\section{Introduction}

Let $\left(Z_{i}\right)_{i \in \mathbb{Z}}$ be a sequence of i.i.d. regularly varying random variables with index of regular variation $\alpha \in(0,2)$. This means that

$$
\mathrm{P}\left(\left|Z_{i}\right|>x\right)=x^{-\alpha} L(x), \quad x>0,
$$

where $L$ is a slowly varying function at $\infty$. Regular variation implies $\mathrm{E}\left|Z_{i}\right|^{\beta}<\infty$ for every $\beta \in(0, \alpha)$. We study the moving average process with random coefficients, defined by

$$
X_{i}=\sum_{j=0}^{\infty} C_{j} Z_{i-j}, \quad i \in \mathbb{Z},
$$

where $\left(C_{i}\right)_{i \geq 0}$ is a sequence of random variables independent of $\left(Z_{i}\right)$, such that the series in (1.2) is a.s. convergent. One sufficient condition for that is

$$
\sum_{j=0}^{\infty}\left|C_{j}\right|^{\alpha-\epsilon}<\infty \quad \text { a.s. for some } \epsilon>0
$$

Received by the editors August 22th, 2018; accepted April 26th, 2019.

2010 Mathematics Subject Classification. 60F17, 60G51.

Key words and phrases. Functional limit theorem, Regular variation, $M_{2}$ topology, Moving Average Process.

Research supported in part by Croatian Science Foundation under the project 3526 and by University of Rijeka under the projects 13.14.1.2.02 and uniri-prirod-18-9. 
(see Hult and Samorodnitsky, 2008). We will use the following moment condition on the sequence $\left(C_{j}\right)$ :

$$
\sum_{j=0}^{\infty} \mathrm{E}\left|C_{j}\right|^{\delta}<\infty \quad \text { for some } \delta<\alpha, 0<\delta \leq 1 .
$$

This condition also implies the a.s. convergence of the series in (1.2), since

$$
\mathrm{E}\left|X_{i}\right|^{\delta} \leq \sum_{j=0}^{\infty} \mathrm{E}\left|C_{j}\right|^{\delta} \mathrm{E}\left|Z_{i-j}\right|^{\delta}=\mathrm{E}\left|Z_{1}\right|^{\delta} \sum_{j=0}^{\infty} \mathrm{E}\left|C_{j}\right|^{\delta}<\infty .
$$

Beside condition (1.4) we will require some other moment conditions, which will be specified in Section 3. We also impose the following (usual) regularity conditions on $Z_{1}$ :

$$
\begin{aligned}
\mathrm{E} Z_{1}=0, & \text { if } \alpha \in(1,2), \\
Z_{1} \text { is symmetric, } & \text { if } \alpha=1 .
\end{aligned}
$$

Let $\left(a_{n}\right)$ be a sequence of positive real numbers such that

$$
n \mathrm{P}\left(\left|Z_{1}\right|>a_{n}\right) \rightarrow 1
$$

as $n \rightarrow \infty$. Regular variation of $Z_{i}$ can be expressed in terms of vague convergence of measures on $\mathbb{E}=\overline{\mathbb{R}} \backslash\{0\}$ : for $a_{n}$ as in (1.7) and as $n \rightarrow \infty$,

$$
n \mathrm{P}\left(a_{n}^{-1} Z_{i} \in \cdot\right) \stackrel{v}{\rightarrow} \mu(\cdot),
$$

with the measure $\mu$ on $\mathbb{E}$ given by

$$
\mu(\mathrm{d} x)=\left(p 1_{(0, \infty)}(x)+r 1_{(-\infty, 0)}(x)\right) \alpha|x|^{-\alpha-1} \mathrm{~d} x,
$$

where

$$
p=\lim _{x \rightarrow \infty} \frac{\mathrm{P}\left(Z_{i}>x\right)}{\mathrm{P}\left(\left|Z_{i}\right|>x\right)} \quad \text { and } \quad r=\lim _{x \rightarrow \infty} \frac{\mathrm{P}\left(Z_{i} \leq-x\right)}{\mathrm{P}\left(\left|Z_{i}\right|>x\right)} .
$$

When the coefficients $C_{i}$ are deterministic, Basrak and Krizmanić (2014) obtained functional convergence of the partial sum process of $X_{i}$ 's with respect to the Skorohod $M_{2}$ topology on $D[0,1]$. More precisely, they showed that under the condition on the coefficients $C_{i}$ :

$$
0 \leq \sum_{i=0}^{s} C_{i} / \sum_{i=0}^{\infty} C_{i} \leq 1, \quad \text { for every } s=0,1,2 \ldots,
$$

the following

$$
\frac{1}{a_{n}} \sum_{i=1}^{\lfloor n \cdot\rfloor} X_{i} \stackrel{d}{\rightarrow}\left(\sum_{j=0}^{\infty} C_{j}\right) V(\cdot)
$$

holds in $D[0,1]$, where $V(\cdot)$ is an $\alpha$-stable Lévy process and $D[0,1]$ is the space of real-valued right continuous functions on $[0,1]$ with left limits.

Recall here that if at least two coefficients are nonzero, then the convergence in (1.12) cannot hold with respect to the more usual Skorohod $J_{1}$ topology on $D[0,1]$, but if all the coefficients are nonnegative, then the convergence in (1.12) holds in the $M_{1}$ topology, see Avram and Taqqu (1992). The aim of this article is to obtain the functional convergence with respect to the $M_{2}$ topology as in (1.12) when the coefficients $C_{i}$ are random variables. Limit theory for moving averages with random coefficients, but without the time component, have already been studied, see Kulik (2006). These processes can represent various stochastic models, such as 
solutions to stochastic recurrence equations and stochastic integrals (usually with some predictability assumption instead of the independence between the coefficients $C_{j}$ and the noise variables $Z_{j}$, see Hult and Samorodnitsky, 2008).

The Skorohod $M_{2}$ topology on $D[0,1]$ is defined using completed graphs and their parametric representations (see Section 12.11 in Whitt, 2002 for details). Here we give only a characterization of the $M_{2}$ topology using the Hausdorff metric on the spaces of graphs, since it will be convenient for our purposes. For $x \in D[0,1]$ the completed graph of $x$ is the set

$$
\Gamma_{x}=\{(t, z) \in[0,1] \times \mathbb{R}: z=\lambda x(t-)+(1-\lambda) x(t) \text { for some } \lambda \in[0,1]\},
$$

where $x(t-)$ is the left limit of $x$ at $t$. Besides the points of the graph $\{(t, x(t))$ : $t \in[0,1]\}$, the graph $\Gamma_{x}$ also contains the vertical line segments joining $(t, x(t))$ and $(t, x(t-))$ for all discontinuity points $t$ of $x$. Now, for $x_{1}, x_{2} \in D[0,1]$ define

$$
d_{M_{2}}\left(x_{1}, x_{2}\right)=\left(\sup _{a \in \Gamma_{x_{1}}} \inf _{b \in \Gamma_{x_{2}}} d(a, b)\right) \vee\left(\sup _{a \in \Gamma_{x_{2}}} \inf _{b \in \Gamma_{x_{1}}} d(a, b)\right),
$$

where $d$ is the metric on $\mathbb{R}^{2}$ defined by $d\left(\left(x_{1}, y_{1}\right),\left(x_{2}, y_{2}\right)\right)=\left|x_{1}-x_{2}\right| \vee\left|y_{1}-y_{2}\right|$ for $\left(x_{i}, y_{i}\right) \in \mathbb{R}^{2}, i=1,2$, where $a \vee b=\max \{a, b\}$. The metric $d_{M_{2}}$ induces the $M_{2}$ topology. This topology is weaker than the more frequently used $M_{1}$ and $J_{1}$ topologies. Therefore the $M_{1}$ convergence implies the $M_{2}$ convergence, but the converse does not hold in general. For instance, take the moving average process with heavy-tailed innovations $Z_{i}$ and deterministic coefficients $C_{0}=1, C_{1}=-1$, $C_{2}=1$, and $C_{i}=0$ for $i \geq 3$, i.e.

$$
X_{i}=Z_{i}-Z_{i-1}+Z_{i-2}, \quad i \in \mathbb{Z} .
$$

Since the condition (1.11) is satisfied, the $M_{2}$ convergence in relation (1.12) holds. Clusters of large values in the sequence $\left(X_{n}\right)$ contain positive and negative values, which means that the corresponding partial sum processes have jumps of opposite signs within temporal clusters of large values, and this precludes the $M_{1}$ convergence. The detailed proof of this fact for the moving average process $\left(X_{i}\right)$ defined above is given in Appendix.

The paper is organized as follows. In Section 2 we obtain functional convergence for finite order moving average processes, and then in Section 3 we extend this result to infinite order moving averages. A technical result needed for establishing functional convergence for infinite order moving averages when $\alpha \in[1,2)$ is given in Appendix.

\section{Finite order MA processes}

Let $C_{0}, C_{1}, \ldots, C_{q}$ (for some fixed $q \in \mathbb{N}$ ) be random variables satisfying

$$
0 \leq \sum_{i=0}^{s} C_{i} / \sum_{i=0}^{q} C_{i} \leq 1 \text { a.s. } \quad \text { for every } s=0,1, \ldots, q .
$$

Put $C=\sum_{i=0}^{q} C_{i}$. Observe that condition (2.1) implies that $C, \sum_{i=0}^{s} C_{i}$ and $\sum_{i=s}^{q} C_{i}$ are a.s. of the same sign for every $s=0,1, \ldots, q$. Also note that condition (2.1) is satisfied if the $C_{j}{ }^{\prime}$ are all nonnegative or all nonpositive. 
Let $\left(X_{t}\right)$ be a moving average process defined by

$$
X_{t}=\sum_{i=0}^{q} C_{i} Z_{t-i}, \quad t \in \mathbb{Z},
$$

and let the corresponding partial sum process be

$$
V_{n}(t)=\frac{1}{a_{n}} \sum_{i=1}^{\lfloor n t\rfloor} X_{i}, \quad t \in[0,1],
$$

where the normalizing sequence $\left(a_{n}\right)$ satisfies $(1.7)$.

Theorem 2.1. Let $\left(Z_{i}\right)_{i \in \mathbb{Z}}$ be an i.i.d. sequence of regularly varying random variables with index $\alpha \in(0,2)$, such that (1.5) and (1.6) hold. Assume $C_{0}, C_{1}, \ldots, C_{q}$ are random variables, independent of $\left(Z_{i}\right)$, that satisfy $(2.1)$. Then

$$
V_{n}(\cdot) \stackrel{d}{\rightarrow} \widetilde{C} V(\cdot), \quad n \rightarrow \infty
$$

in $D[0,1]$ endowed with the $M_{2}$ topology, where $V$ is an $\alpha$-stable Lévy process with characteristic triple $(0, \mu, b)$, with $\mu$ as in (1.9) and

$$
b=\left\{\begin{array}{cc}
0, & \alpha=1, \\
(p-r) \frac{\alpha}{1-\alpha}, & \alpha \in(0,1) \cup(1,2),
\end{array}\right.
$$

and $\widetilde{C}$ is a random variable, independent of $V$, such that $\widetilde{C} \stackrel{d}{=} C$.

As in Basrak and Krizmanić (2014) one can prove the following lemma (with the notation $C_{i}=0$ for $\left.i<0\right)$.

Lemma 2.2. $\quad$ (i) For $k<q$ it holds

$$
\begin{aligned}
\sum_{i=1}^{k} \frac{C Z_{i}}{a_{n}}-\sum_{i=1}^{k} \frac{X_{i}}{a_{n}}= & \sum_{u=0}^{k-1} \frac{Z_{k-u}}{a_{n}} \sum_{s=u+1}^{q} C_{s}-\sum_{u=k-q}^{q-1} \frac{Z_{-u}}{a_{n}} \sum_{s=u+1}^{q} C_{s} \\
& -\sum_{u=0}^{q-k-1} \frac{Z_{-u}}{a_{n}} \sum_{s=u+1}^{u+k} C_{s} .
\end{aligned}
$$

(ii) For $k \geq q$ it holds

$$
\begin{aligned}
\sum_{i=1}^{k} \frac{C Z_{i}}{a_{n}}-\sum_{i=1}^{k} \frac{X_{i}}{a_{n}} & =\sum_{u=0}^{q-1} \frac{Z_{k-u}}{a_{n}} \sum_{s=u+1}^{q} C_{s}-\sum_{u=0}^{q-1} \frac{Z_{-u}}{a_{n}} \sum_{s=u+1}^{q} C_{s} \\
& =: \quad H_{n}(k)-G_{n} .
\end{aligned}
$$

(iii) For $q \leq k \leq n-q$ it holds

$$
\begin{aligned}
\sum_{i=1}^{k} \frac{C Z_{i}}{a_{n}}-\sum_{i=1}^{k+q} \frac{X_{i}}{a_{n}} & =-\sum_{u=0}^{q-1} \frac{Z_{-u}}{a_{n}} \sum_{s=u+1}^{q} C_{s}-\sum_{u=1}^{q} \frac{Z_{k+u}}{a_{n}} \sum_{s=0}^{q-u} C_{s} \\
& =: \quad-G_{n}-T_{n}(k) .
\end{aligned}
$$


Proof: (Theorem 2.1) Since the random variables $Z_{i}$ are i.i.d. and regularly varying, it is known that

$$
\sum_{i=1}^{\lfloor n t\rfloor} \frac{Z_{i}}{a_{n}}-\lfloor n t\rfloor \mathrm{E}\left(\frac{Z_{1}}{a_{n}} 1_{\left\{\left|Z_{1}\right| \leq a_{n}\right\}}\right), \quad t \in[0,1],
$$

converges in distribution, as $n \rightarrow \infty$, in $D[0,1]$ with the $M_{1}$ topology to an $\alpha$-stable Lévy process with characteristic triple $(0, \mu, 0)$ (see Theorem 3.4 in Basrak et al., 2012). By Karamata's theorem, as $n \rightarrow \infty$,

$$
\begin{aligned}
& n \mathrm{E}\left(\frac{Z_{1}}{a_{n}} 1_{\left\{\left|Z_{1}\right| \leq a_{n}\right\}}\right) \rightarrow(p-r) \frac{\alpha}{1-\alpha}, \quad \text { if } \alpha<1, \\
& n \mathrm{E}\left(\frac{Z_{1}}{a_{n}} 1_{\left\{\left|Z_{1}\right|>a_{n}\right\}}\right) \rightarrow(p-r) \frac{\alpha}{\alpha-1}, \quad \text { if } \quad \alpha>1,
\end{aligned}
$$

with $p$ and $r$ as in (1.10). Therefore conditions (1.5) and (1.6), Corollary 12.7.1 in Whitt (2002) (which gives a sufficient condition for addition to be continuous in the $M_{1}$ topology) and the continuous mapping theorem yield that $V_{n}^{Z}(\cdot) \stackrel{d}{\rightarrow} V(\cdot)$, as $n \rightarrow \infty$, in $D[0,1]$ with the $M_{1}$ topology, where

$$
V_{n}^{Z}(t):=\sum_{i=1}^{\lfloor n t\rfloor} \frac{Z_{i}}{a_{n}}, \quad t \in[0,1],
$$

and $V$ is an $\alpha$-stable Lévy process with characteristic triple $(0, \mu, b)$.

It is well known that the space $D[0,1]$ equipped with the Skorohod $J_{1}$ topology is a Polish space (i.e. metrizable as a complete separable metric space), see Billingsley (1968), Section 14. The same holds for the $M_{1}$ topology, since it is topologically complete (see Whitt, 2002, Section 12.8) and separability remains preserved in the weaker topology. Therefore by Corollary 5.18 in Kallenberg (1997), we can find a random variable $\widetilde{C}$, independent of $V$, such that $\widetilde{C} \stackrel{d}{=} C$. This and the fact that $C$ is independent of $V_{n}^{Z}$, by an application of Theorem 3.29 in Kallenberg (1997), imply

$$
\left(B(\cdot), V_{n}^{Z}(\cdot)\right) \stackrel{d}{\rightarrow}(\widetilde{B}(\cdot), V(\cdot)), \quad \text { as } n \rightarrow \infty,
$$

in $D\left([0,1], \mathbb{R}^{2}\right)$ with the product $M_{1}$ topology, where $B(t)=C$ and $\widetilde{B}(t)=\widetilde{C}$ for $t \in[0,1]$.

Let $g: D\left([0,1], \mathbb{R}^{2}\right) \rightarrow D[0,1]$ be a function defined by

$$
g(x)=x_{1} x_{2}, \quad x=\left(x_{1}, x_{2}\right) \in D\left([0,1], \mathbb{R}^{2}\right),
$$

where $\left(x_{1} x_{2}\right)(t)=x_{1}(t) x_{2}(t)$ for $t \in[0,1]$. Let

$$
D_{1}=\{u \in D([0,1]: \operatorname{Disc}(u)=\emptyset\},
$$

and

$$
D_{2}=\left\{(u, v) \in D\left([0,1], \mathbb{R}^{2}\right): \operatorname{Disc}(u)=\emptyset\right\},
$$

where $\operatorname{Disc}(u)$ is the set of discontinuity points of $u$. Then by Theorem 13.3.2 in Whitt (2002) the function $g$ is continuous on the set $D_{2}$ (with the Skorohod $M_{1}$ topology on $D[0,1]$ and product $M_{1}$ topology on $\left.D\left([0,1], \mathbb{R}^{2}\right)\right)$. Hence $\operatorname{Disc}(g) \subseteq D_{2}^{c}$, and

$$
\mathrm{P}[(\widetilde{B}, V) \in \operatorname{Disc}(g)] \leq \mathrm{P}\left[(\widetilde{B}, V) \in D_{2}^{c}\right] \leq \mathrm{P}\left(\widetilde{B} \in D_{1}^{c}\right)=0 .
$$


This allows us to apply the continuous mapping theorem (see for instance Theorem 3.1 in Resnick, 2007) to relation (2.3) which yields $g\left(B, V_{n}^{Z}\right) \stackrel{d}{\rightarrow} g(\widetilde{B}, V)$, i.e.

$$
C V_{n}^{Z}(\cdot) \stackrel{d}{\rightarrow} \widetilde{C} V(\cdot), \quad \text { as } n \rightarrow \infty,
$$

in $D[0,1]$ with the $M_{1}$ topology. Using the fact that $M_{1}$ convergence implies $M_{2}$ convergence, we obtain

$$
C V_{n}^{Z}(\cdot) \stackrel{d}{\rightarrow} \widetilde{C} V(\cdot), \quad \text { as } n \rightarrow \infty,
$$

in $\left(D[0,1], d_{M_{2}}\right)$ as well. If we can show that for every $\epsilon>0$

$$
\lim _{n \rightarrow \infty} \mathrm{P}\left[d_{M_{2}}\left(C V_{n}^{Z}, V_{n}\right)>\epsilon\right]=0,
$$

an application of Slutsky's theorem (see for instance Theorem 3.4 in Resnick, 2007) will imply $V_{n}(\cdot) \stackrel{d}{\rightarrow} \widetilde{C} V(\cdot)$, as $n \rightarrow \infty$, in $\left(D[0,1], d_{M_{2}}\right)$.

Fix $\epsilon>0$ and let $n \in \mathbb{N}$ be large enough, i.e. $n>\max \{2 q, 2 q / \epsilon\}$. By the definition of the metric $d_{M_{2}}$ we have

$$
\begin{aligned}
d_{M_{2}}\left(C V_{n}^{Z}, V_{n}\right) & =\left(\sup _{a \in \Gamma_{C V_{n}^{Z}}} \inf _{b \in \Gamma_{V_{n}}} d(a, b)\right) \vee\left(\sup _{a \in \Gamma_{V_{n}}} \inf _{b \in \Gamma_{C V_{n}^{Z}}} d(a, b)\right) \\
& =: \quad Y_{n} \vee T_{n},
\end{aligned}
$$

and therefore

$$
\mathrm{P}\left[d_{M_{2}}\left(V_{n}^{Z}, V_{n}\right)>\epsilon\right] \leq \mathrm{P}\left(Y_{n}>\epsilon\right)+\mathrm{P}\left(T_{n}>\epsilon\right) .
$$

In order to estimate the first term on the right hand side of (2.5) note that

$$
\begin{aligned}
\left\{Y_{n}>\epsilon\right\} \subseteq & \left\{\exists a \in \Gamma_{C V_{n}^{Z}} \text { such that } d(a, b)>\epsilon \text { for every } b \in \Gamma_{V_{n}}\right\} \\
\subseteq \quad & \left\{\exists k \in\{1, \ldots, q-1\} \text { such that }\left|C V_{n}^{Z}(k / n)-V_{n}(k / n)\right|>\epsilon\right\} \\
& \cup\left\{\exists k \in\{q, \ldots, n-q\} \text { such that }\left|C V_{n}^{Z}(k / n)-V_{n}(k / n)\right|>\epsilon\right. \\
& \text { and } \left.\left|C V_{n}^{Z}(k / n)-V_{n}((k+q) / n)\right|>\epsilon\right\} \\
& \cup\left\{\exists k \in\{n-q+1, \ldots, n\} \text { such that }\left|C V_{n}^{Z}(k / n)-V_{n}(k / n)\right|>\epsilon\right\} \\
=: & A_{n}^{Y} \cup B_{n}^{Y} \cup C_{n}^{Y},
\end{aligned}
$$

where the second inclusion above follows from the fact that the paths of $V_{n}$ and $C V_{n}^{Z}$ are constant on the intervals of the form

$$
\left[\frac{j}{n}, \frac{j+1}{n}\right), \quad j=0,1, \ldots, n-1 .
$$

More precisely, if there is a point $a=\left(t_{a}, x_{a}\right) \in \Gamma_{C V_{n}^{Z}}$ such that $d\left(a, \Gamma_{V_{n}}\right)>\epsilon$, then necessarily $t_{a} \in[i / n,(i+1) / n)$ for some $i=1, \ldots, n$. If $a$ lies on a horizontal part of the completed graph, then $x_{a}=C V_{n}^{Z}(i / n)$ and

$$
\left|C V_{n}^{Z}(i / n)-V_{n}(i / n)\right| \geq d\left(a, \Gamma_{V_{n}}\right)>\epsilon .
$$

Alternatively, if $a$ lies on a vertical part of the completed graph, then $x_{a} \in\left[C V_{n}^{Z}((i-\right.$ 1) $\left./ n), C V_{n}^{Z}(i / n)\right)$, and one can similarly conclude that

$$
\left|C V_{n}^{Z}(k / n)-V_{n}(k / n)\right|>\epsilon
$$


for some $k=1, \ldots, n$ (in fact $k=i$ or $k=i-1$; see Basrak and Krizmanić, 2014 for details). Moreover, if $q \leq k \leq n-q$, from $q / n<\epsilon / 2$ it follows similarly that

$$
\left|C V_{n}^{Z}(k / n)-V_{n}((k+q) / n)\right|>\epsilon .
$$

By Lemma 2.2 (i) we obtain

$$
\begin{aligned}
\mathrm{P}\left(A_{n}^{Y}\right) \leq & \sum_{k=1}^{q-1} \mathrm{P}\left(\left|\sum_{i=1}^{k} \frac{C Z_{i}}{a_{n}}-\sum_{i=1}^{k} \frac{X_{i}}{a_{n}}\right|>\epsilon\right) \\
\leq & \sum_{k=1}^{q-1}\left[\mathrm{P}\left(\sum_{u=0}^{k-1} \frac{\left|Z_{k-u}\right|}{a_{n}} \sum_{s=u+1}^{q}\left|C_{s}\right|>\frac{\epsilon}{3}\right)+\mathrm{P}\left(\sum_{u=k-q}^{q-1} \frac{\left|Z_{-u}\right|}{a_{n}} \sum_{s=u+1}^{q}\left|C_{s}\right|>\frac{\epsilon}{3}\right)\right. \\
& \left.+\mathrm{P}\left(\sum_{u=0}^{q-k-1} \frac{\left|Z_{-u}\right|}{a_{n}} \sum_{s=u+1}^{u+k}\left|C_{s}\right|>\frac{\epsilon}{3}\right)\right] \\
\leq & 3(q-1)(2 q-1) \mathrm{P}\left(\frac{\left|Z_{0}\right|}{a_{n}} C_{*}>\frac{\epsilon}{3(2 q-1)}\right),
\end{aligned}
$$

where $C_{*}=\sum_{s=0}^{q}\left|C_{s}\right|$. For an arbitrary $M>0$ it holds that

$$
\begin{aligned}
& \mathrm{P}\left(\frac{\left|Z_{0}\right|}{a_{n}} C_{*}>\frac{\epsilon}{3(2 q-1)}\right) \\
& =\mathrm{P}\left(\frac{\left|Z_{0}\right|}{a_{n}} C_{*}>\frac{\epsilon}{3(2 q-1)}, C_{*}>M\right)+\mathrm{P}\left(\frac{\left|Z_{0}\right|}{a_{n}} C_{*}>\frac{\epsilon}{3(2 q-1)}, C_{*} \leq M\right) \\
& \leq \mathrm{P}\left(C_{*}>M\right)+\mathrm{P}\left(\frac{\left|Z_{0}\right|}{a_{n}}>\frac{\epsilon}{3(2 q-1) M}\right) .
\end{aligned}
$$

By the regular variation property we observe

$$
\lim _{n \rightarrow \infty} \mathrm{P}\left(\frac{\left|Z_{0}\right|}{a_{n}}>\frac{\epsilon}{3(2 q-1) M}\right)=0,
$$

and hence from (2.7) we get

$$
\limsup _{n \rightarrow \infty} \mathrm{P}\left(A_{n}^{Y}\right) \leq \mathrm{P}\left(C_{*}>M\right) .
$$

Letting $M \rightarrow \infty$ we conclude

$$
\lim _{n \rightarrow \infty} \mathrm{P}\left(A_{n}^{Y}\right)=0 .
$$

Next, using Lemma 2.2 (ii) and (iii), for an arbitrary $M>0$ we obtain

$$
\begin{gathered}
\mathrm{P}\left(B_{n}^{Y} \cap\left\{C_{*} \leq M\right\}\right)=\mathrm{P}\left(\exists k \in\{q, \ldots, n-q\} \text { such that }\left|H_{n}(k)-G_{n}\right|>\epsilon\right. \\
\text { and } \left.\left|-G_{n}-T_{n}(k)\right|>\epsilon, C_{*} \leq M\right) \\
\leq \mathrm{P}\left(\left|G_{n}\right|>\frac{\epsilon}{2}, C_{*} \leq M\right)+\sum_{k=q}^{n-q} \mathrm{P}\left(\left|H_{n}(k)\right|>\frac{\epsilon}{2} \text { and }\left|T_{n}(k)\right|>\frac{\epsilon}{2}, C_{*} \leq M\right) .
\end{gathered}
$$


Note that

$$
\begin{aligned}
\mathrm{P}\left(\left|G_{n}\right|>\frac{\epsilon}{2}, C_{*} \leq M\right) & \leq \mathrm{P}\left(C_{*} \sum_{u=0}^{q-1} \frac{\left|Z_{-u}\right|}{a_{n}}>\frac{\epsilon}{2}, C_{*} \leq M\right) \\
& \leq \mathrm{P}\left(\sum_{u=0}^{q-1} \frac{\left|Z_{-u}\right|}{a_{n}}>\frac{\epsilon}{2 M}\right) \\
& \leq q \mathrm{P}\left(\frac{\left|Z_{0}\right|}{a_{n}}>\frac{\epsilon}{2 q M}\right) .
\end{aligned}
$$

Similarly

$$
\begin{aligned}
& \mathrm{P}\left(\left|H_{n}(k)\right|>\frac{\epsilon}{2}\right. \text { and }\left.\left|T_{n}(k)\right|>\frac{\epsilon}{2}, C_{*} \leq M\right) \\
& \leq \mathrm{P}\left(\sum_{u=0}^{q-1} \frac{\left|Z_{k-u}\right|}{a_{n}}>\frac{\epsilon}{2 M} \text { and } \sum_{u=1}^{q} \frac{\left|Z_{k+u}\right|}{a_{n}}>\frac{\epsilon}{2 M}\right) \\
&=\mathrm{P}\left(\sum_{u=0}^{q-1} \frac{\left|Z_{k-u}\right|}{a_{n}}>\frac{\epsilon}{2 M}\right) \mathrm{P}\left(\sum_{u=1}^{q} \frac{\left|Z_{k+u}\right|}{a_{n}}>\frac{\epsilon}{2 M}\right) \\
& \leq\left[q \mathrm{P}\left(\frac{\left|Z_{0}\right|}{a_{n}}>\frac{\epsilon}{2 q M}\right)\right]^{2},
\end{aligned}
$$

where the equality above holds since the random variables $Z_{i}$ are independent. Therefore

$$
\begin{aligned}
\mathrm{P}\left(B_{n}^{Y} \cap\left\{C_{*} \leq M\right\}\right) & \leq q \mathrm{P}\left(\frac{\left|Z_{0}\right|}{a_{n}}>\frac{\epsilon}{2 q M}\right)+\sum_{k=q}^{n-q}\left[q \mathrm{P}\left(\frac{\left|Z_{0}\right|}{a_{n}}>\frac{\epsilon}{2 q M}\right)\right]^{2} \\
& \leq q \mathrm{P}\left(\frac{\left|Z_{0}\right|}{a_{n}}>\frac{\epsilon}{2 q M}\right)+\frac{q^{2}}{n}\left[n \mathrm{P}\left(\frac{\left|Z_{0}\right|}{a_{n}}>\frac{\epsilon}{2 q M}\right)\right]^{2}
\end{aligned}
$$

and an application of the regular variation property yields

$$
\lim _{n \rightarrow \infty} \mathrm{P}\left(B_{n}^{Y} \cap\left\{C_{*} \leq M\right\}\right)=0
$$

Thus

$$
\limsup _{n \rightarrow \infty} \mathrm{P}\left(B_{n}^{Y}\right) \leq \limsup _{n \rightarrow \infty} \mathrm{P}\left(B_{n}^{Y} \cap\left\{C_{*}>M\right\}\right) \leq \mathrm{P}\left(C_{*}>M\right),
$$

and letting again $M \rightarrow \infty$ we conclude

$$
\lim _{n \rightarrow \infty} \mathrm{P}\left(B_{n}^{Y}\right)=0 .
$$

In a similar manner as in (2.7), but using (ii) from Lemma 2.2 instead of (i) we get

$$
\lim _{n \rightarrow \infty} \mathrm{P}\left(C_{n}^{Y}\right)=0 \text {. }
$$

From relations (2.6), (2.8), (2.9) and (2.10) we obtain

$$
\lim _{n \rightarrow \infty} \mathrm{P}\left(Y_{n}>\epsilon\right)=0 \text {. }
$$

It remains to estimate the second term on the right hand side of (2.5). For each $k \geq q$, set $V_{k}^{Z, \min }=\min \left\{C V_{n}^{Z}((k-q) / n), C V_{n}^{Z}(k / n)\right\}$ and $V_{k}^{Z, \max }=\max \{$ 
$\left.C V_{n}^{Z}((k-q) / n), C V_{n}^{Z}(k / n)\right\}$. From the definition of $T_{n}$, the Hausdorff metric and the number $n$ it follows

$$
\begin{aligned}
\left\{T_{n}>\epsilon\right\} \subseteq & \left\{\exists a \in \Gamma_{V_{n}} \text { such that } d(a, b)>\epsilon \text { for every } b \in \Gamma_{C V_{n}^{Z}}\right\} \\
\subseteq & \left\{\exists k \in\{1, \ldots, 2 q-1\} \text { such that }\left|V_{n}(k / n)-C V_{n}^{Z}(k / n)\right|>\epsilon\right\} \\
& \cup\left\{\exists k \in\{2 q, \ldots, n\} \text { such that } \widetilde{d}\left(V_{n}(k / n),\left[V_{k}^{Z, \min }, V_{k}^{Z, \max }\right]\right)>\epsilon\right\} \\
=: & A_{n}^{T} \cup B_{n}^{T},
\end{aligned}
$$

where $\widetilde{d}$ is the Euclidean metric on $\mathbb{R}$. The argument behind the second inclusion in (2.12) is similar to the one given after (2.6). Indeed, assume there is a point $a=\left(t_{a}, x_{a}\right) \in \Gamma_{V_{n}}$ such that

$$
d\left(a, \Gamma_{C V_{n}^{Z}}\right)>\epsilon
$$

Then necessarily $t_{a} \in[i / n,(i+1) / n)$ for some $i=1, \ldots, n$. The case $i \leq 2 q-1$ is covered by the same argument used to obtain (2.6) and the set $A_{n}^{Y}$. Therefore, we may assume $i \geq 2 q$. From (2.13) we immediately obtain

$$
d\left(a,\left(i / n, C V_{n}^{Z}(i / n)\right)\right)>\epsilon \quad \text { and } \quad d\left(a,\left((i-q) / n, C V_{n}^{Z}((i-q) / n)\right)\right)>\epsilon .
$$

Suppose first that $x_{a}=V_{n}(i / n)$ for some $i=2 q, \ldots, n$. Recall that $q / n<\epsilon / 2$. Since $\max \left\{\left|t_{a}-i / n\right|,\left|t_{a}-(i-q) / n\right|\right\} \leq(q+1) / n<\epsilon$, from (2.14) we conclude that

$$
\widetilde{d}\left(V_{n}(i / n),\left[V_{i}^{Z, \min }, V_{i}^{Z, \max }\right]\right)>\epsilon .
$$

If $x_{a} \in\left[V_{n}((i-1) / n), V_{n}(i / n)\right)$ (in this case $\left.t_{a}=i / n\right)$, relation (2.14) again implies $\widetilde{d}\left(x_{a},\left[V_{i}^{Z, \min }, V_{i}^{Z, \max }\right]\right)>\epsilon$, and similarly $\widetilde{d}\left(x_{a},\left[V_{i-1}^{Z, \min }, V_{i-1}^{Z, \max }\right]\right)>\epsilon$. Thus we obtain

$$
\max \left\{\tilde{d}\left(V_{n}(i / n),\left[V_{i}^{Z, \min }, V_{i}^{Z, \max }\right]\right), \widetilde{d}\left(V_{n}((i-1) / n),\left[V_{i-1}^{Z, \min }, V_{i-1}^{Z, \max }\right]\right)\right\}>\epsilon .
$$

Finally we conclude that there exists $k \in\{2 q, \ldots, n\}$ such that

$$
\widetilde{d}\left(V_{n}(k / n),\left[V_{k}^{Z, \min }, V_{k}^{Z, \max }\right]\right)>\epsilon .
$$

Using Lemma 2.2 (i) and (ii), one could similarly as before for the set $A_{n}^{Y}$ obtain

$$
\lim _{n \rightarrow \infty} \mathrm{P}\left(A_{n}^{T}\right)=0 .
$$

Note that $\mathrm{P}\left(B_{n}^{T}\right)$ is bounded above by

$$
\begin{aligned}
& \mathrm{P}\left(\exists k \in\{2 q, \ldots, n\} \text { such that } \sum_{i=1}^{k} \frac{X_{i}}{a_{n}}>V_{k}^{Z, \max }+\epsilon\right) \\
& \quad+\mathrm{P}\left(\exists k \in\{2 q, \ldots, n\} \text { such that } \sum_{i=1}^{k} \frac{X_{i}}{a_{n}}<V_{k}^{Z, \min }-\epsilon\right) .
\end{aligned}
$$

In the sequel we consider only the first of these two probabilities, since the other one can be handled in a similar manner. The first probability using Lemma 2.2 can be bounded by

$$
\begin{aligned}
\mathrm{P}(\exists k & \left.\in\{2 q, \ldots, n\} \text { such that } G_{n}-H_{n}(k)>\epsilon \text { and } G_{n}+T_{n}(k-q)>\epsilon\right) \\
\leq & \mathrm{P}\left(G_{n}>\frac{\epsilon}{2}\right) \\
& +\mathrm{P}\left(\exists k \in\{2 q, \ldots, n\} \text { such that } H_{n}(k)<-\frac{\epsilon}{2} \text { and } T_{n}(k-q)>\frac{\epsilon}{2}\right) .
\end{aligned}
$$


From the calculations yielding (2.9) we conclude that $\mathrm{P}\left(G_{n}>\epsilon / 2\right) \rightarrow 0$ as $n \rightarrow \infty$. The second term is bounded by

$$
\mathrm{P}\left(C_{*}>M\right)+\sum_{k=2 q}^{n} \mathrm{P}\left(H_{n}(k)<-\frac{\epsilon}{2} \text { and } T_{n}(k-q)>\frac{\epsilon}{2}, C_{*} \leq M\right)
$$

for an arbitrary $M>0$. Note that

$$
H_{n}(k)=\sum_{u=0}^{q-1} \frac{Z_{k-u}}{a_{n}} \sum_{s=u+1}^{q} C_{s} \text { and } T_{n}(k-q)=\sum_{u=0}^{q-1} \frac{Z_{k-u}}{a_{n}} \sum_{s=0}^{u} C_{s} .
$$

Therefore for a fixed $k \in\{2 q, \ldots, n\}$, on the event $\left\{H_{n}(k)<-\epsilon / 2\right.$ and $T_{n}(k-q)>$ $\left.\epsilon / 2, C_{*} \leq M\right\}$ there exist $i, j \in\{0, \ldots, q-1\}$ such that

$$
\frac{Z_{k-i}}{a_{n}} \sum_{s=i+1}^{q} C_{s}<-\frac{\epsilon}{2 q} \quad \text { and } \quad \frac{Z_{k-j}}{a_{n}} \sum_{s=0}^{j} C_{s}>\frac{\epsilon}{2 q} \text {. }
$$

From (2.1) it follows that the sums $\sum_{s=0}^{j} C_{s}$ and $\sum_{s=i+1}^{q} C_{s}$ are a.s. of the same sign and their absolute values are bounded by $C_{*}$. Hence if these sums are positive we obtain $Z_{k-i} M / a_{n}<-\epsilon /(2 q)$ and $Z_{k-j} M / a_{n}>\epsilon /(2 q)$, while if they are negative we obtain $Z_{k-i} M / a_{n}>\epsilon /(2 q)$ and $Z_{k-j} M / a_{n}<-\epsilon /(2 q)$. Note that the case $i=j$ is not possible since then we would have $Z_{k-i}<0$ and $Z_{k-i}>0$. From this, using the stationarity of the sequence $\left(Z_{i}\right)$, we conclude that the expression in $(2.16)$ is bounded by

$$
\begin{aligned}
& \mathrm{P}\left(C_{*}>M\right)+n \mathrm{P}\left(\exists i, j \in\{0, \ldots, q-1\}, i \neq j \text { s.t. } M \frac{Z_{-i}}{a_{n}}<-\frac{\epsilon}{2 q} \text { and } M \frac{Z_{-j}}{a_{n}}>\frac{\epsilon}{2 q}\right) \\
& \leq \mathrm{P}\left(C_{*}>M\right)+n\left(\begin{array}{l}
q \\
2
\end{array}\right)\left[\mathrm{P}\left(\frac{\left|Z_{0}\right|}{a_{n}}>\frac{\epsilon}{2 q M}\right)\right]^{2},
\end{aligned}
$$

which tends to 0 if we first let $n \rightarrow \infty$ and then $M \rightarrow \infty$. Together with relations (2.12) and (2.15) this implies

$$
\lim _{n \rightarrow \infty} \mathrm{P}\left(T_{n}>\epsilon\right)=0 .
$$

Now from (2.5), (2.11) and (2.17) we obtain

$$
\lim _{n \rightarrow \infty} \mathrm{P}\left[d_{M_{2}}\left(C V_{n}^{Z}, V_{n}\right)>\epsilon\right]=0,
$$

and finally we conclude that $V_{n}(\cdot) \stackrel{d}{\rightarrow} \widetilde{C} V(\cdot)$, as $n \rightarrow \infty$, in $\left(D[0,1], d_{M_{2}}\right)$. This concludes the proof.

\section{Infinite order MA processes}

Let $\left(X_{i}\right)$ be a moving average process defined by

$$
X_{i}=\sum_{j=0}^{\infty} C_{j} Z_{i-j}, \quad i \in \mathbb{Z},
$$

where $\left(Z_{i}\right)$ is an i.i.d. sequence of regularly varying random variables with index $\alpha \in(0,2)$, such that $\mathrm{E} Z_{i}=0$ if $\alpha \in(1,2)$ and $Z_{i}$ is symmetric if $\alpha=1$. Let $\left\{C_{i}, i=\right.$ 
$0,1,2, \ldots\}$ be a sequence of random variables, independent of $\left(Z_{i}\right)$, satisfying

$$
\sum_{i=0}^{\infty} \mathrm{E}\left|C_{i}\right|^{\delta}<\infty \quad \text { for some } \delta<\alpha, 0<\delta \leq 1,
$$

and

$$
0 \leq \sum_{i=0}^{s} C_{i} / \sum_{i=0}^{\infty} C_{i} \leq 1 \text { a.s. } \quad \text { for every } s=0,1,2 \ldots .
$$

Let $C=\sum_{i=0}^{\infty} C_{i}$. Condition (3.1) implies $C$ is a.s. finite, and ensures that the series in the definition of $X_{i}$ above converges almost surely. Define further the corresponding partial sum stochastic process $V_{n}$ as in (2.2). Beside the above stated conditions, we require also the following conditions: for $\alpha \in(0,1)$

$$
\sum_{i=0}^{\infty} \mathrm{E}\left|C_{i}\right|^{\gamma}<\infty \quad \text { for some } \gamma \in(\alpha, 1)
$$

and for $\alpha \in[1,2)$

$$
\lim _{n \rightarrow \infty}(\ln n)^{1+\eta} \mathrm{E}\left[\left(\sum_{i=n}^{\infty}\left|C_{i}\right|\right)^{\eta-\delta} \sum_{j=n}^{\infty}\left|C_{j}\right|^{\delta}\right]=0 \quad \text { for some } \eta>\alpha .
$$

The latter condition is borrowed from Avram and Taqqu (1992), where they studied $M_{1}$ functional convergence of sums of moving averages with deterministic coefficients. Since in the case $\alpha \in(1,2)$ we will also need that the series $\sum_{i=1}^{\infty} \mathrm{E}\left|C_{i}\right|$ converges, we assume $\delta=1$ in (3.1) if $\alpha>1$.

For a deterministic sequence $\left(C_{j}\right)$ condition (3.3) is not needed since it is implied by (3.1). The latter in general does not hold when the coefficients $C_{j}$ are random. It can easily be seen by the following example. Take $\epsilon>0$ such that $\delta+\epsilon<\gamma$. Let $S=\sum_{j=1}^{\infty} j^{-(1+\delta+\epsilon)}<\infty$ and $S_{k}=S^{-1} \sum_{j=1}^{k} j^{-(1+\delta+\epsilon)}, k \in \mathbb{N}\left(\right.$ with $\left.S_{0}=0\right)$. Taking $\mathrm{P}$ to be the Lebesgue measure on the Borel subsets of $(0,1)$ and

$$
C_{i}(\omega)=i 1_{\left(S_{i-1}, S_{i}\right]}(\omega), \quad \omega \in(0,1), i \in \mathbb{N},
$$

we obtain

$$
\sum_{i=1}^{\infty} \mathrm{E}\left|C_{i}\right|^{\delta}=S^{-1} \sum_{i=1}^{\infty} i^{\delta}\left(S_{i}-S_{i-1}\right)=S^{-1} \sum_{i=1}^{\infty} \frac{1}{i^{1+\epsilon}}<\infty
$$

and

$$
\sum_{i=1}^{\infty} \mathrm{E}\left|C_{i}\right|^{\gamma}=S^{-1} \sum_{i=1}^{\infty} i^{\gamma}\left(S_{i}-S_{i-1}\right)=S^{-1} \sum_{i=1}^{\infty} \frac{1}{i^{1+\delta+\epsilon-\gamma}}=\infty,
$$

since $1+\delta+\epsilon-\gamma<1$.

Theorem 3.1. Let $\left(Z_{i}\right)_{i \in \mathbb{Z}}$ be an i.i.d. sequence of regularly varying random variables with index $\alpha \in(0,2)$. Suppose that conditions (1.5) and (1.6) hold. Let $\left\{C_{i}, i=0,1,2, \ldots\right\}$ be a sequence of random variables, independent of $\left(Z_{i}\right)$, such that (3.1) and (3.2) hold. Assume also (3.3) holds if $\alpha \in(0,1)$, and (3.4) if $\alpha \in[1,2)$. Then

$$
V_{n}(\cdot) \stackrel{d}{\rightarrow} \widetilde{C} V(\cdot), \quad n \rightarrow \infty
$$


in $D[0,1]$ endowed with the $M_{2}$ topology, where $V$ is an $\alpha$-stable Lévy process with characteristic triple $(0, \mu, b)$, with $\mu$ as in (1.9) and

$$
b=\left\{\begin{array}{cc}
0, & \alpha=1, \\
(p-r) \frac{\alpha}{1-\alpha}, & \alpha \in(0,1) \cup(1,2),
\end{array}\right.
$$

and $\widetilde{C}$ is a random variable, independent of $V$, such that $\widetilde{C} \stackrel{d}{=} C$.

In the proof of the theorem we are going to use the following three lemmas, in which $\left(Z_{i}\right)_{i \in \mathbb{Z}}$ is an i.i.d. sequence of regularly varying random variables with index $\alpha,\left(C_{i}\right)_{i \geq 0}$ is a sequence of random variables, independent of $\left(Z_{i}\right)$, and $\left(a_{n}\right)$ is a sequence of positive real numbers satisfying (1.7).

Lemma 3.2. Let $\alpha \in(0,1)$, and assume conditions (3.1) and (3.3) hold. Then for every $\epsilon>0$

$$
\lim _{q \rightarrow \infty} \limsup _{n \rightarrow \infty} \mathrm{P}\left[\left(2 \sum_{j=q+1}^{\infty}\left|C_{j}\right|\right) \sum_{i=1}^{n} \frac{\left|Z_{i-q}\right|}{a_{n}}+\sum_{i=-\infty}^{0} \frac{\left|Z_{i-q}\right|}{a_{n}} \sum_{j=1}^{n}\left|C_{q-i+j}\right|>\epsilon\right]=0 .
$$

Proof: Let

$$
D_{i}^{n, q}= \begin{cases}2 \sum_{j=q+1}^{\infty}\left|C_{j}\right|, & i=1, \ldots, n \\ \sum_{j=1}^{n}\left|C_{q-i+j}\right|, & i \leq 0 .\end{cases}
$$

We have to show

$$
\lim _{q \rightarrow \infty} \limsup _{n \rightarrow \infty} \mathrm{P}\left(\sum_{i=-\infty}^{n} \frac{D_{i}^{n, q}\left|Z_{i-q}\right|}{a_{n}}>\epsilon\right)=0
$$

Let

$$
Z_{i, n}^{\leq}=\frac{Z_{i}}{a_{n}} 1_{\left\{\frac{\left|Z_{i}\right|}{a_{n}} \leq 1\right\}} \quad \text { and } \quad Z_{i, n}^{>}=\frac{Z_{i}}{a_{n}} 1_{\left\{\frac{\left|Z_{i}\right|}{a_{n}}>1\right\}},
$$

and note that the probability in (3.5) is bounded above by

$$
\mathrm{P}\left(\sum_{i=-\infty}^{n} D_{i}^{n, q}\left|Z_{i-q, n}^{\leq}\right|>\frac{\epsilon}{2}\right)+\mathrm{P}\left(\sum_{i=-\infty}^{n} D_{i}^{n, q}\left|Z_{i-q, n}^{>}\right|>\frac{\epsilon}{2}\right) .
$$

Using Markov's inequality, the triangle inequality $\left|\sum_{i=1}^{\infty} a_{i}\right|^{s} \leq \sum_{i=1}^{\infty}\left|a_{i}\right|^{s}$ with $s \in(0,1]$, the fact that $\left(C_{i}\right)$ is independent of $\left(Z_{i}\right)$ and the stationarity of the sequence $\left(Z_{i}\right)$, for the first term in (3.6) we obtain

$$
\begin{aligned}
\mathrm{P}\left(\sum_{i=-\infty}^{n} D_{i}^{n, q}\left|Z_{i-q, n}^{\leq}\right|>\frac{\epsilon}{2}\right) & \leq\left(\frac{\epsilon}{2}\right)^{-\gamma} \mathrm{E}\left(\sum_{i=-\infty}^{n} D_{i}^{n, q}\left|Z_{i-q, n}^{\leq}\right|\right)^{\gamma} \\
& \leq\left(\frac{\epsilon}{2}\right)^{-\gamma} \mathrm{E}\left(\sum_{i=-\infty}^{n}\left(D_{i}^{n, q}\right)^{\gamma}\left|Z_{i-q, n}^{\leq}\right|^{\gamma}\right) \\
& \leq\left(\frac{\epsilon}{2}\right)^{-\gamma} \mathrm{E}\left|Z_{1, n}^{\leq}\right|^{\gamma} \sum_{i=-\infty}^{n} \mathrm{E}\left(D_{i}^{n, q}\right)^{\gamma} .
\end{aligned}
$$


Again by triangle inequality we have

$$
\sum_{i=-\infty}^{n} \mathrm{E}\left(D_{i}^{n, q}\right)^{\gamma} \leq 2^{\gamma} n \sum_{j=q+1}^{\infty} \mathrm{E}\left|C_{j}\right|^{\gamma}+\sum_{i=-\infty}^{0} \sum_{j=1}^{n} \mathrm{E}\left|C_{q-i+j}\right|^{\gamma},
$$

Note that every $\mathrm{E}\left|C_{j}\right|^{\gamma}$, for $j=q+1, q+2, \ldots$, appears in the sum $\sum_{i=-\infty}^{0} \sum_{j=1}^{n} \mathrm{E}\left|C_{q-i+j}\right|^{\gamma}$ at most $n$ times, and hence

$$
\begin{aligned}
\mathrm{P}\left(\sum_{i=-\infty}^{n} D_{i}^{n, q}\left|Z_{i-q, n}^{\leq}\right|>\frac{\epsilon}{2}\right) & \leq\left(\frac{\epsilon}{2}\right)^{-\gamma} \mathrm{E}\left|Z_{1, n}^{\leq}\right|^{\gamma}\left(2^{\gamma} n \sum_{j=q+1}^{\infty} \mathrm{E}\left|C_{j}\right|^{\gamma}+n \sum_{j=q+1}^{\infty} \mathrm{E}\left|C_{j}\right|^{\gamma}\right) \\
& =\left(2^{\gamma}+1\right)\left(\frac{\epsilon}{2}\right)^{-\gamma} n \mathrm{E}\left|Z_{1, n}^{\leq}\right|^{\gamma} \sum_{j=q+1}^{\infty} \mathrm{E}\left|C_{j}\right|^{\gamma} .
\end{aligned}
$$

Similarly

$$
\mathrm{P}\left(\sum_{i=-\infty}^{n} D_{i}^{n, q}\left|Z_{i-q, n}^{>}\right|>\frac{\epsilon}{2}\right) \leq\left(2^{\delta}+1\right)\left(\frac{\epsilon}{2}\right)^{-\delta} n \mathrm{E}\left|Z_{1, n}^{>}\right|^{\delta} \sum_{j=q+1}^{\infty} \mathrm{E}\left|C_{j}\right|^{\delta} .
$$

By Karamata's theorem and (1.7), as $n \rightarrow \infty$,

$$
n \mathrm{E}\left|Z_{1, n}^{\leq}\right|^{\gamma}=\frac{\mathrm{E}\left(\left|Z_{1}\right|^{\gamma} 1_{\left\{\left|Z_{1}\right| \leq a_{n}\right\}}\right)}{a_{n}^{\gamma} \mathrm{P}\left(\left|Z_{1}\right|>a_{n}\right)} \cdot n \mathrm{P}\left(\left|Z_{1}\right|>a_{n}\right) \rightarrow \frac{\alpha}{\gamma-\alpha}<\infty
$$

and

$$
n \mathrm{E}\left|Z_{1, n}^{>}\right|^{\delta}=\frac{\mathrm{E}\left(\left|Z_{1}\right|{ }^{\delta} 1_{\left\{\left|Z_{1}\right|>a_{n}\right\}}\right)}{a_{n}^{\delta} \mathrm{P}\left(\left|Z_{1}\right|>a_{n}\right)} \cdot n \mathrm{P}\left(\left|Z_{1}\right|>a_{n}\right) \rightarrow \frac{\alpha}{\alpha-\delta}<\infty .
$$

From this and relations (3.7) and (3.8) we conclude that

$$
\limsup _{n \rightarrow \infty} \mathrm{P}\left(\sum_{i=-\infty}^{n} \frac{D_{i}^{n, q}\left|Z_{i-q}\right|}{a_{n}}>\epsilon\right) \leq M\left(\sum_{j=q+1}^{\infty} \mathrm{E}\left|C_{j}\right|^{\gamma}+\sum_{j=q+1}^{\infty} \mathrm{E}\left|C_{j}\right|^{\delta}\right),
$$

where $M=\left(2^{\gamma}+1\right)(\epsilon / 2)^{-\gamma} \alpha /(\gamma-\alpha)+\left(2^{\delta}+1\right)(\epsilon / 2)^{-\delta} \alpha /(\alpha-\delta)<\infty$. Now letting $q \rightarrow \infty$, conditions (3.1) and (3.3) imply (3.5).

Lemma 3.3. Let $\alpha \in(1,2)$. Assume conditions (3.1) and (3.4) hold, and $\mathrm{E} Z_{1}=0$. If $\left(q_{n}\right)$ is a sequence of positive integers tending to infinity, then

$$
\lim _{n \rightarrow \infty} \mathrm{P}\left(\sup _{0 \leq t \leq 1}\left|\sum_{i=1}^{\lfloor n t\rfloor} \frac{C_{q_{n}}^{\prime \prime} Z_{i-q_{n}}}{a_{n}}\right|>\epsilon\right)=0 \quad \text { for every } \epsilon>0,
$$

where $C_{q_{n}}^{\prime \prime}=\sum_{j=q_{n}+1}^{\infty} C_{j}$.

Proof: Let

$$
\widetilde{Z}_{i, n}^{\leq}=Z_{i, n}^{\leq}-\mathrm{E} Z_{i, n}^{\leq} \quad \text { and } \quad \widetilde{Z}_{i, n}^{>}=Z_{i, n}^{>}+\mathrm{E} Z_{i, n}^{\leq},
$$

and note that $Z_{i} / a_{n}=\widetilde{Z}_{i, n}^{\leq}+\widetilde{Z}_{i, n}^{>}, \mathrm{E} \widetilde{Z}_{i, n}^{\leq}=0$ and also $\mathrm{E} \widetilde{Z}_{i, n}^{>}=\mathrm{E} Z_{i, n}^{>}+\mathrm{E} Z_{i, n}^{\leq}=$ $\mathrm{E}\left(Z_{i} / a_{n}\right)=0$. Thus

$$
\mathrm{P}\left(\sup _{0 \leq t \leq 1}\left|\sum_{i=1}^{\lfloor n t\rfloor} \frac{C_{q_{n}}^{\prime \prime} Z_{i-q_{n}}}{a_{n}}\right|>\epsilon\right)
$$




$$
\begin{aligned}
& \leq \mathrm{P}\left(\sup _{1 \leq k \leq n}\left|\sum_{i=1}^{k} C_{q_{n}}^{\prime \prime} \widetilde{Z}_{i-q_{n}, n}^{\leq}\right|>\frac{\epsilon}{2}\right)+\mathrm{P}\left(\sup _{1 \leq k \leq n}\left|\sum_{i=1}^{k} C_{q_{n}}^{\prime \prime} \widetilde{Z}_{i-q_{n}, n}^{>}\right|>\frac{\epsilon}{2}\right) \\
& =: I_{1}+I_{2} .
\end{aligned}
$$

Since $C_{q_{n}}^{\prime \prime}$ is independent of $\left(Z_{i}\right)$ and $\mathrm{E} \widetilde{Z}_{i, n}^{\leq}=0$, it follows that $\left(\sum_{i=1}^{k} C_{q_{n}}^{\prime \prime} \widetilde{Z}_{i-q_{n}, n}^{\leq}\right)_{k}$ is a martingale (with respect to the filtration $\left(\mathcal{F}_{k}\right)$, where the $\sigma$-field $\mathcal{F}_{k}$ is generated by $C_{i}, i \geq 0$ and $\left.Z_{j-q_{n}}, j \leq k-q_{n}\right)$. Hence by Markov's inequality and Doob's maximal inequality

$$
\mathrm{E}\left(\sup _{1 \leq k \leq n}\left|S_{k}\right|\right)^{\kappa} \leq\left(\frac{\kappa}{\kappa-1}\right)^{\kappa} \mathrm{E}\left|S_{n}\right|^{\kappa}
$$

which holds for $\kappa>1$ and $\left(S_{k}\right)_{k}$ a martingale (see Durrett, 1996, p. 251) we obtain

$$
I_{1} \leq\left(\frac{\epsilon}{2}\right)^{-\eta}\left(\frac{\eta}{\eta-1}\right)^{\eta} \mathrm{E}\left|\sum_{i=1}^{n} C_{q_{n}}^{\prime \prime} \widetilde{Z}_{i-q_{n}, n}^{\leq}\right|^{\eta}
$$

with $\eta$ as in (3.4). Note that $\left(C_{q_{n}}^{\prime \prime} \widetilde{Z}_{i-q_{n}, n}^{\leq}\right)_{i}$ is a martingale difference sequence, and hence by the Bahr-Esseen inequality

$$
\mathrm{E}\left|\sum_{j=1}^{n} Y_{j}\right|^{\kappa} \leq 2 \sum_{j=1}^{n} \mathrm{E}\left|Y_{j}\right|^{\kappa}
$$

which holds for $\kappa \in[1,2]$ and $\left(Y_{j}\right)_{j}$ a martingale-difference sequence (see Chatterji, 1969, Lemma 1) we have

$$
\begin{aligned}
I_{1} & \leq 2\left(\frac{\epsilon}{2}\right)^{-\eta}\left(\frac{\eta}{\eta-1}\right)^{\eta} \sum_{i=1}^{n} \mathrm{E}\left|C_{q_{n}}^{\prime \prime} \widetilde{Z}_{i-q_{n}, n}^{\leq}\right|^{\eta} \\
& =2\left(\frac{\epsilon}{2}\right)^{-\eta}\left(\frac{\eta}{\eta-1}\right)^{\eta} \mathrm{E}\left|\widetilde{Z}_{1, n}^{\leq}\right|^{\eta} \sum_{i=1}^{n} \mathrm{E}\left|C_{q_{n}}^{\prime \prime}\right|^{\eta} \\
& =2\left(\frac{\epsilon}{2}\right)^{-\eta}\left(\frac{\eta}{\eta-1}\right)^{\eta} n \mathrm{E}\left|\widetilde{Z}_{1, n}^{\leq}\right|^{\eta} \mathrm{E}\left|C_{q_{n}}^{\prime \prime}\right|^{\eta} .
\end{aligned}
$$

Using the inequality $|a-b|^{\eta} \leq 2^{\eta}\left(|a|^{\eta}+|b|^{\eta}\right)$ and a special case of Jensen's inequality

$$
(\mathrm{E}|Y|)^{\kappa} \leq \mathrm{E}|Y|^{\kappa}
$$

(which holds for $\kappa \geq 1$ ) we have

$$
\mathrm{E}\left|\widetilde{Z}_{1, n}^{\leq}\right|^{\eta} \leq 2^{\eta}\left[\mathrm{E}\left|Z_{1, n}^{\leq}\right|^{\eta}+\left(\mathrm{E}\left|Z_{1, n}^{\leq}\right|\right)^{\eta}\right] \leq 2^{\eta+1} \mathrm{E}\left|Z_{1, n}^{\leq}\right|^{\eta},
$$

and hence

Note that

$$
I_{1} \leq 2^{2+2 \eta} \epsilon^{-\eta}\left(\frac{\eta}{\eta-1}\right)^{\eta} n \mathrm{E}\left|Z_{1, n}^{\leq}\right|^{\eta} \mathrm{E}\left|C_{q_{n}}^{\prime \prime}\right|^{\eta}
$$

$$
\mathrm{E}\left|C_{q_{n}}^{\prime \prime}\right|^{\eta}=\mathrm{E}\left(\left|C_{q_{n}}^{\prime \prime}\right|^{\eta-\delta} \cdot\left|C_{q_{n}}^{\prime \prime}\right|^{\delta}\right) \leq \mathrm{E}\left[\left(\sum_{i=q_{n}+1}^{\infty}\left|C_{i}\right|\right)^{\eta-\delta} \sum_{j=q_{n}+1}^{\infty}\left|C_{j}\right|^{\delta}\right],
$$

and thus condition (3.4) yields $\lim _{n \rightarrow \infty} \mathrm{E}\left|C_{q_{n}}^{\prime \prime}\right|^{\eta}=0$. This and the fact that $\lim _{n \rightarrow \infty} n \mathrm{E}\left|Z_{1, n}^{\leq}\right|^{\eta}=\alpha /(\eta-\alpha)$ (which holds by Karamata's theorem) allows us to conclude that $\lim _{n \rightarrow \infty} I_{1}=0$. 
For $I_{2}$ by Markov's inequality we obtain

$$
I_{2} \leq \mathrm{P}\left(\sum_{i=1}^{n}\left|C_{q_{n}}^{\prime \prime} \widetilde{Z}_{i-q_{n}, n}^{>}\right|>\frac{\epsilon}{2}\right) \leq\left(\frac{\epsilon}{2}\right)^{-1} \mathrm{E}\left|\widetilde{Z}_{1, n}^{>}\right| \sum_{i=1}^{n} \mathrm{E}\left|C_{q_{n}}^{\prime \prime}\right| .
$$

Since $\widetilde{Z}_{i, n}^{>}=Z_{i, n}^{>}-\mathrm{E} Z_{i, n}^{>}$, it holds that

$$
\mathrm{E}\left|\widetilde{Z}_{1, n}^{>}\right| \leq \mathrm{E}\left|Z_{1, n}^{>}\right|+\left|\mathrm{E} Z_{1, n}^{>}\right| \leq 2 \mathrm{E}\left|Z_{1, n}^{>}\right|
$$

Therefore

$$
I_{2} \leq 4 \epsilon^{-1} n \mathrm{E}\left|Z_{1, n}^{>}\right| \sum_{j=q_{n}+1}^{\infty} \mathrm{E}\left|C_{j}\right|
$$

yielding $\lim _{n \rightarrow \infty} I_{2}=0$, since by Karamata's theorem $\lim _{n \rightarrow \infty} n \mathrm{E}\left|Z_{1, n}^{>}\right|=\alpha /(\alpha-1)$ and we assumed (3.1) holds with $\delta=1$ in this case. This completes the proof of the lemma.

Lemma 3.4. Let $\alpha \in(1,2)$. Assume conditions (3.1) and (3.4) hold, and $\mathrm{E} Z_{1}=$ 0 . If $\left(q_{n}\right)$ is a sequence of positive integers tending to infinity, such that $\ln n=$ $O\left(\ln q_{n}\right)$, then

$$
\lim _{n \rightarrow \infty} \mathrm{P}\left(\sup _{0 \leq t \leq 1}\left|\sum_{i=1}^{\lfloor n t\rfloor} \sum_{j=q_{n}+1}^{\infty} \frac{C_{j} Z_{i-j}}{a_{n}}\right|>\epsilon\right)=0 \quad \text { for every } \epsilon>0 .
$$

Proof: Note that

$$
\begin{aligned}
& \mathrm{P}\left(\sup _{0 \leq t \leq 1}\left|\sum_{i=1}^{\lfloor n t\rfloor} \sum_{j=q_{n}+1}^{\infty} \frac{C_{j} Z_{i-j}}{a_{n}}\right|>\epsilon\right) \\
& \leq \mathrm{P}\left(\sup _{0 \leq t \leq 1}\left|\sum_{i=1}^{\lfloor n t\rfloor} \sum_{j=q_{n}+1}^{\infty} C_{j} \widetilde{Z}_{i-j, n}^{\leq}\right|>\frac{\epsilon}{2}\right)+\mathrm{P}\left(\sup _{0 \leq t \leq 1}\left|\sum_{i=1}^{\lfloor n t\rfloor} \sum_{j=q_{n}+1}^{\infty} C_{j} \widetilde{Z}_{i-j, n}^{>}\right|>\frac{\epsilon}{2}\right) \\
& =: I_{3}+I_{4} .
\end{aligned}
$$

Let

$$
W_{n}(t)=\sum_{i=1}^{\lfloor n t\rfloor} \sum_{j=q_{n}+1}^{\infty} C_{j} \widetilde{Z}_{i-j, n}^{\leq}, \quad t \in[0,1] .
$$

Take $0 \leq t_{1}<t_{2} \leq 1$, and consider (for $\rho>0$ )

$$
\begin{aligned}
\mathrm{P}\left(\left|W_{n}\left(t_{2}\right)-W_{n}\left(t_{1}\right)\right|>\rho\right) & =\mathrm{P}\left(\left|\sum_{i=\left\lfloor n t_{1}\right\rfloor+1}^{\left\lfloor n t_{2}\right\rfloor} \sum_{j=q_{n}+1}^{\infty} C_{j} \widetilde{Z}_{i-j, n}^{\leq}\right|>\rho\right) \\
& =\mathrm{P}\left(\left|\sum_{i=-\infty}^{\left\lfloor n t_{2}\right\rfloor-1} \widetilde{D}_{i-q_{n}}^{n, t_{1}, t_{2}} \widetilde{Z}_{i-q_{n}, n}^{\leq}\right|>\rho\right),
\end{aligned}
$$


where

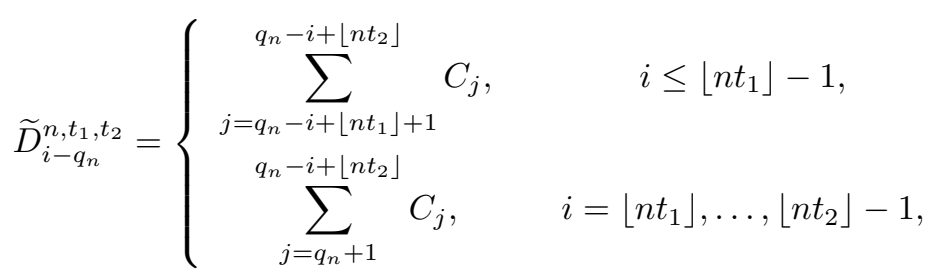

and the last equality in (3.12) follows by standard changes of variables and order of summation. The sequence $\left(\widetilde{D}_{i-q_{n}}^{n, t_{1}, t_{2}} \widetilde{Z}_{i-q_{n}, n}^{\leq}\right)_{i}$ is a martingale difference sequence, and hence the Bahr-Esseen inequality (which holds also for infinite sums, by the Fatou lemma) and Markov's inequality imply

$$
\begin{aligned}
\mathrm{P}\left(\left|\sum_{i=-\infty}^{\left\lfloor n t_{2}\right\rfloor-1} \widetilde{D}_{i-q_{n}}^{n, t_{1}, t_{2}} \widetilde{Z}_{i-q_{n}, n}^{\leq}\right|>\rho\right) & \leq \rho^{-\eta}\left(\frac{\eta}{\eta-1}\right)^{\eta} \sum_{i=-\infty}^{\left\lfloor n t_{2}\right\rfloor-1} \mathrm{E}\left|\widetilde{D}_{i-q_{n}}^{n, t_{1}, t_{2}} \widetilde{Z}_{i-q_{n}, n}^{\leq}\right|^{\eta} \\
& =\rho^{-\eta}\left(\frac{\eta}{\eta-1}\right)^{\eta} \mathrm{E}\left|\widetilde{Z}_{1, n}^{\leq}\right|^{\eta} \sum_{i=-\infty}^{\left\lfloor n t_{2}\right\rfloor-1} \mathrm{E}\left|\widetilde{D}_{i-q_{n}}^{n, t_{1}, t_{2}}\right|^{\eta},
\end{aligned}
$$

with $\eta$ as in (3.4). With the same argument as in (3.10) we obtain

$$
\begin{aligned}
& \sum_{i=\left\lfloor n t_{1}\right\rfloor}^{\left\lfloor n t_{2}\right\rfloor-1} \mathrm{E}\left|\widetilde{D}_{i-q_{n}}^{n, t_{1}, t_{2}}\right|^{\eta} \\
& \quad \leq \sum_{i=\left\lfloor n t_{1}\right\rfloor}^{\left\lfloor n t_{2}\right\rfloor-1} \mathrm{E}\left[\left(\sum_{s=q_{n}+1}^{\infty}\left|C_{s}\right|\right)^{\eta-\delta} \sum_{j=q_{n}+1}^{\infty}\left|C_{j}\right|^{\delta}\right] \\
& \quad=\left(\left\lfloor n t_{2}\right\rfloor-\left\lfloor n t_{1}\right\rfloor\right) \mathrm{E}\left[\left(\sum_{s=q_{n}+1}^{\infty}\left|C_{s}\right|\right)^{\eta-\delta} \sum_{j=q_{n}+1}^{\infty}\left|C_{j}\right|^{\delta}\right]
\end{aligned}
$$

and

$$
\begin{aligned}
& \sum_{i=-\infty}^{\left\lfloor n t_{1}\right\rfloor-1} \mathrm{E}\left|\widetilde{D}_{i-q_{n}}^{n, t_{1}, t_{2}}\right|^{\eta} \\
& \quad \leq \mathrm{E}\left[\left(\sum_{s=q_{n}+1}^{\infty}\left|C_{s}\right|\right)^{\eta-\delta} \sum_{i=-\infty}^{\left\lfloor n t_{1}\right\rfloor-1} \sum_{j=q_{n}-i+\left\lfloor n t_{1}\right\rfloor+1}^{q_{n}-i+\left\lfloor n t_{2}\right\rfloor}\left|C_{j}\right|^{\delta}\right] \\
& \leq\left(\left\lfloor n t_{2}\right\rfloor-\left\lfloor n t_{1}\right\rfloor\right) \mathrm{E}\left[\left(\sum_{s=q_{n}+1}^{\infty}\left|C_{s}\right|\right)^{\eta-\delta} \sum_{j=q_{n}+1}^{\infty}\left|C_{j}\right|^{\delta}\right]
\end{aligned}
$$

where the last inequality follows from the fact that every $\left|C_{j}\right|^{\delta}$, for $j \geq q_{n}+1$, appears in the sum $\sum_{i=-\infty}^{\left\lfloor n t_{1}\right\rfloor-1} \sum_{j=q_{n}-i+\left\lfloor n t_{1}\right\rfloor+1}^{q_{n}-i+\left\lfloor n t_{2}\right\rfloor}\left|C_{j}\right|^{\delta}$ at most $\left\lfloor n t_{2}\right\rfloor-\left\lfloor n t_{1}\right\rfloor$ times. Therefore

$$
\mathrm{P}\left(\left|\sum_{i=-\infty}^{\left\lfloor n t_{2}\right\rfloor-1} \widetilde{D}_{i-q_{n}}^{n, t_{1}, t_{2}} \widetilde{Z}_{i-q_{n}, n}^{\leq}\right|>\rho\right)
$$




$$
\leq 2 \rho^{-\eta}\left(\frac{\eta}{\eta-1}\right)^{\eta} n \mathrm{E}\left|\widetilde{Z}_{1, n}^{\leq}\right|^{\eta} \frac{\left\lfloor n t_{2}\right\rfloor-\left\lfloor n t_{1}\right\rfloor}{n} \mathrm{E}\left[\left(\sum_{s=q_{n}+1}^{\infty}\left|C_{s}\right|\right)^{\eta-\delta} \sum_{j=q_{n}+1}^{\infty}\left|C_{j}\right|^{\delta}\right] .
$$

Since by (3.9) and Karamata's theorem $\sup _{n}\left\{n \mathrm{E}\left|\widetilde{Z}_{1, n}^{\leq}\right|^{\eta}\right\}<\infty$, and $\left(\left\lfloor n t_{2}\right\rfloor-\right.$ $\left.\left\lfloor n t_{1}\right\rfloor\right) / n \leq 2\left(t_{2}-t_{1}\right)$ for large $n$, it follows that

$\mathrm{P}\left(\left|\sum_{i=-\infty}^{\left\lfloor n t_{2}\right\rfloor-1} \widetilde{D}_{i-q_{n}}^{n, t_{1}, t_{2}} \widetilde{Z}_{i-q_{n}, n}^{\leq}\right|>\rho\right) \leq M \rho^{-\eta}\left(t_{2}-t_{1}\right) \mathrm{E}\left[\left(\sum_{s=q_{n}+1}^{\infty}\left|C_{s}\right|\right)^{\eta-\delta} \sum_{j=q_{n}+1}^{\infty}\left|C_{j}\right|^{\delta}\right]$,

for some constant $M$ independent of $n$. Now by Theorem 2 in Avram and Taqqu (1989) and the arguments in the proof of Proposition 4 in Avram and Taqqu (1992) we conclude that

$$
\begin{aligned}
\mathrm{P}\left(\sup _{0 \leq t \leq 1}\left|\sum_{i=-\infty}^{\lfloor n t\rfloor-1} \widetilde{D}_{i-q_{n}}^{n, 0, t} \widetilde{Z}_{i-q_{n}, n}^{\leq}\right|>\rho\right) \\
\leq M^{\prime} \rho^{-\eta}(\ln n)^{1+\eta} \mathrm{E}\left[\left(\sum_{s=q_{n}+1}^{\infty}\left|C_{s}\right|\right)^{\eta-\delta} \sum_{j=q_{n}+1}^{\infty}\left|C_{j}\right|^{\delta}\right]
\end{aligned}
$$

for some constant $M^{\prime}$ independent of $n$. From this and condition (3.4), since $\ln n=$ $O\left(\ln q_{n}\right)$, it follows that

$$
\lim _{n \rightarrow \infty} I_{3}=\lim _{n \rightarrow \infty} \mathrm{P}\left(\sup _{0 \leq t \leq 1}\left|\sum_{i=-\infty}^{\lfloor n t\rfloor-1} \widetilde{D}_{i-q_{n}}^{n, 0, t} \widetilde{Z}_{i-q_{n}, n}^{\leq}\right|>\frac{\epsilon}{2}\right)=0
$$

Further, note that

$$
\begin{aligned}
\mathrm{P}\left(\sup _{0 \leq t \leq 1}\left|\sum_{i=1}^{\lfloor n t\rfloor} \sum_{j=q_{n}+1}^{\infty} C_{j} \widetilde{Z}_{i-j, n}^{>}\right|>\frac{\epsilon}{2}\right) & \leq \mathrm{P}\left(\sum_{i=1}^{n} \sum_{j=q_{n}+1}^{\infty}\left|C_{j} \widetilde{Z}_{i-j, n}^{>}\right|>\frac{\epsilon}{2}\right) \\
& \leq\left(\frac{\epsilon}{2}\right)^{-1} \sum_{i=1}^{n} \sum_{j=q_{n}+1}^{\infty} \mathrm{E}\left|C_{j} \widetilde{Z}_{i-j, n}^{>}\right| \\
& =\left(\frac{\epsilon}{2}\right)^{-1} \mathrm{E}\left|\widetilde{Z}_{1, n}^{>}\right| \sum_{i=1}^{n} \sum_{j=q_{n}+1}^{\infty} \mathrm{E}\left|C_{j}\right| \\
& =\left(\frac{\epsilon}{2}\right)^{-1} n \mathrm{E}\left|\widetilde{Z}_{1, n}^{>}\right| \sum_{j=q_{n}+1}^{\infty} \mathrm{E}\left|C_{j}\right| .
\end{aligned}
$$

By (3.11) and Karamata's theorem $\sup _{n}\left\{n \mathrm{E}\left|\widetilde{Z}_{1, n}^{>}\right|\right\}<\infty$, and hence condition (3.1) (with $\delta=1$ ) implies

$$
\lim _{n \rightarrow \infty} I_{4}=0 \text {. }
$$

Now from (3.15) and (3.16) we get the conclusion of the lemma.

We are now ready to prove Theorem 3.1.

Proof: (Theorem 3.1) For $q \in \mathbb{N}$ define

$$
X_{i}^{q}=\sum_{j=0}^{q-1} C_{j} Z_{i-j}+C_{q}^{\prime} Z_{i-q}, \quad i \in \mathbb{Z}
$$


where $C_{q}^{\prime}=\sum_{i=q}^{\infty} C_{i}$, and

$$
V_{n, q}(t)=\sum_{i=1}^{\lfloor n t\rfloor} \frac{X_{i}^{q}}{a_{n}}, \quad t \in[0,1] .
$$

Now we treat separately the cases $\alpha \in(0,1), \alpha \in(1,2)$ and $\alpha=1$.

Case $\alpha \in(0,1)$. Fix $q \in \mathbb{N}$. Since the coefficients $C_{0}, \ldots, C_{q-1}, C_{q}^{\prime}$ satisfy condition (2.1), an application of Theorem 2.1 to a finite order moving average process $\left(X_{i}^{q}\right)_{i}$ yields that, as $n \rightarrow \infty$,

$$
V_{n, q}(\cdot) \stackrel{d}{\rightarrow} \widetilde{C} V(\cdot)
$$

in $\left(D[0,1], d_{M_{2}}\right)$. If we show that for every $\epsilon>0$

$$
\lim _{q \rightarrow \infty} \limsup _{n \rightarrow \infty} \mathrm{P}\left[d_{M_{2}}\left(V_{n, q}, V_{n}\right)>\epsilon\right]=0,
$$

then by a generalization of Slutsky's theorem (see for instance Theorem 3.5 in Resnick, 2007) it will follow $V_{n}(\cdot) \stackrel{d}{\rightarrow} \widetilde{C} V(\cdot)$, as $n \rightarrow \infty$, in $\left(D[0,1], d_{M_{2}}\right)$. Since the Skorohod $M_{2}$ metric on $D[0,1]$ is bounded above by the uniform metric on $D[0,1]$, it suffices to show that

$$
\lim _{q \rightarrow \infty} \limsup _{n \rightarrow \infty} \mathrm{P}\left(\sup _{0 \leq t \leq 1}\left|V_{n, q}(t)-V_{n}(t)\right|>\epsilon\right)=0 .
$$

Recalling the definitions, we have

$$
\begin{aligned}
\lim _{q \rightarrow \infty} \limsup _{n \rightarrow \infty} \mathrm{P} & \left(\sup _{0 \leq t \leq 1}\left|V_{n, q}(t)-V_{n}(t)\right|>\epsilon\right) \\
& \leq \lim _{q \rightarrow \infty} \limsup _{n \rightarrow \infty} \mathrm{P}\left(\sum_{i=1}^{n} \frac{\left|X_{i}^{q}-X_{i}\right|}{a_{n}}>\epsilon\right) .
\end{aligned}
$$

Put $C_{q}^{\prime \prime}=C_{q}^{\prime}-C_{q}=\sum_{j=q+1}^{\infty} C_{j}$ and observe

$$
\begin{aligned}
\sum_{i=1}^{n}\left|X_{i}^{q}-X_{i}\right| & =\sum_{i=1}^{n}\left|\sum_{j=0}^{q-1} C_{j} Z_{i-j}+C_{q}^{\prime} Z_{i-q}-\sum_{j=0}^{\infty} C_{j} Z_{i-j}\right| \\
& =\sum_{i=1}^{n}\left|C_{q}^{\prime \prime} Z_{i-q}-\sum_{j=q+1}^{\infty} C_{j} Z_{i-j}\right| \\
& \leq \sum_{i=1}^{n}\left[\left|C_{q}^{\prime \prime}\right|\left|Z_{i-q}\right|+\sum_{j=q+1}^{\infty}\left|C_{j}\right|\left|Z_{i-j}\right|\right] \\
& \leq\left(2 \sum_{j=q+1}^{\infty}\left|C_{j}\right|\right) \sum_{i=1}^{n}\left|Z_{i-q}\right|+\sum_{i=-\infty}^{0}\left|Z_{i-q}\right| \sum_{j=1}^{n}\left|C_{q-i+j}\right|
\end{aligned}
$$

Lemma 3.2 now implies

$$
\lim _{q \rightarrow \infty} \limsup _{n \rightarrow \infty} \mathrm{P}\left(\sum_{i=1}^{n} \frac{\left|X_{i}^{q}-X_{i}\right|}{a_{n}}>\epsilon\right)=0,
$$

which means that $V_{n}(\cdot) \stackrel{d}{\rightarrow} \widetilde{C} V(\cdot)$, as $n \rightarrow \infty$, in $\left(D[0,1], d_{M_{2}}\right)$. 
Case $\alpha \in(1,2)$. Let $\left(q_{n}\right)$ be a sequence of positive integers such that $q_{n}=$ $\left\lfloor n^{1 / 10}\right\rfloor$. We first show that $\lim _{n \rightarrow \infty} \mathrm{P}\left[d_{M_{2}}\left(V_{n, q_{n}}, V_{n}\right)>\epsilon\right]=0$ for every $\epsilon>0$. For this, similar to the case $\alpha \in(0,1)$, it suffices to show that

$$
\lim _{n \rightarrow \infty} \mathrm{P}\left(\sup _{0 \leq t \leq 1}\left|V_{n, q_{n}}(t)-V_{n}(t)\right|>\epsilon\right)=0 .
$$

Recalling the definitions, we have

$$
V_{n, q_{n}}(t)-V_{n}(t)=\frac{1}{a_{n}} \sum_{i=1}^{\lfloor n t\rfloor}\left(X_{i}^{q_{n}}-X_{i}\right)=\frac{1}{a_{n}} \sum_{i=1}^{\lfloor n t\rfloor}\left(C_{q_{n}}^{\prime \prime} Z_{i-q_{n}}+\sum_{j=q_{n}+1}^{\infty} C_{j} Z_{i-j}\right),
$$

and hence

$$
\begin{aligned}
& \mathrm{P}\left(\sup _{0 \leq t \leq 1}\left|V_{n, q_{n}}(t)-V_{n}(t)\right|>\epsilon\right) \\
& \leq \mathrm{P}\left(\sup _{0 \leq t \leq 1}\left|\sum_{i=1}^{\lfloor n t\rfloor} \frac{C_{q_{n}}^{\prime \prime} Z_{i-q_{n}}}{a_{n}}\right|>\frac{\epsilon}{2}\right)+\mathrm{P}\left(\sup _{0 \leq t \leq 1}\left|\sum_{i=1}^{\lfloor n t\rfloor} \sum_{j=q_{n}+1}^{\infty} \frac{C_{j} Z_{i-j}}{a_{n}}\right|>\frac{\epsilon}{2}\right) .
\end{aligned}
$$

Applying Lemma 3.3 and Lemma 3.4 we conclude

$$
\lim _{n \rightarrow \infty} \mathrm{P}\left(\sup _{0 \leq t \leq 1}\left|V_{n, q_{n}}(t)-V_{n}(t)\right|>\epsilon\right)=0 .
$$

Thus, in order to have $V_{n}(\cdot) \stackrel{d}{\rightarrow} \widetilde{C} V(\cdot)$ in $D[0,1]$ with the $M_{2}$ topology, according to Slutsky's theorem (see Resnick, 2007, Theorem 3.4), it remains to show $V_{n, q_{n}}(\cdot) \stackrel{d}{\rightarrow} \widetilde{C} V(\cdot)$ in $\left(D[0,1], d_{M_{2}}\right)$ as $n \rightarrow \infty$. Note that we cannot simply use Theorem 2.1 as we did in the case $\alpha \in(0,1)$, since now $q_{n}$ depends on $n$. By careful analysis of the proof of Theorem 2.1 we see that relations that have to be checked, in order that the statement of Theorem 2.1 remains valid if we replace $q$ by $q_{n}$, are (2.8), (2.9) and (2.17) (with $C_{*}=\sum_{s=0}^{\infty}\left|C_{s}\right|$ ). Hence we have to establish the following relations

$$
\begin{aligned}
& \lim _{n \rightarrow \infty}\left(q_{n}-1\right)\left(2 q_{n}-1\right) \mathrm{P}\left(\frac{\left|Z_{0}\right|}{a_{n}}>\frac{\epsilon}{3\left(2 q_{n}-1\right) M}\right)=0 \\
& \lim _{n \rightarrow \infty}\left[q_{n} \mathrm{P}\left(\frac{\left|Z_{0}\right|}{a_{n}}>\frac{\epsilon}{2 q_{n} M}\right)+n q_{n}^{2}\left(\mathrm{P}\left(\frac{\left|Z_{0}\right|}{a_{n}}>\frac{\epsilon}{2 q_{n} M}\right)\right)^{2}\right]=0 \\
& \lim _{n \rightarrow \infty} n\left(\begin{array}{c}
q_{n} \\
2
\end{array}\right)\left[\mathrm{P}\left(\frac{\left|Z_{0}\right|}{a_{n}}>\frac{\epsilon}{2 q_{n} M}\right)\right]^{2}=0,
\end{aligned}
$$

for arbitrary $\epsilon>0$ and $M>0$. For all of this, taking into consideration relation (1.1), i.e. the regular variation property of $Z_{0}$, it suffices to show

$$
\lim _{n \rightarrow \infty} n q_{n}^{2}\left[\mathrm{P}\left(\left|Z_{0}\right|>\frac{a_{n}}{q_{n}}\right)\right]^{2}=0
$$

which holds by Lemma 4.1 in Appendix. Therefore we conclude $V_{n}(\cdot) \stackrel{d}{\rightarrow} \widetilde{C} V(\cdot)$ in $\left(D[0,1], d_{M_{2}}\right)$.

Case $\alpha=1$. Since $Z_{i}$ is symmetric, note that $\widetilde{Z}_{i, n}^{\leq}=Z_{i, n}^{\leq}$and $\widetilde{Z}_{i, n}^{>}=Z_{i, n}^{>}$. We proceed as in the case $\alpha \in(1,2)$ to obtain $\lim _{n \rightarrow \infty} I_{1}=0$ and $\lim _{n \rightarrow \infty} I_{3}=0$ (with 
the notation form the proofs of Lemma 3.3 and Lemma 3.4). For $I_{2}$, by Markov's inequality and the triangle inequality $\left|\sum_{i=1}^{n} a_{i}\right|^{\gamma} \leq \sum_{i=1}^{n}\left|a_{i}\right|^{\delta}$ we obtain

$$
I_{2} \leq \mathrm{P}\left(\sum_{i=1}^{n}\left|C_{q_{n}}^{\prime \prime} \widetilde{Z}_{i-q_{n}, n}^{>}\right|>\frac{\epsilon}{2}\right) \leq\left(\frac{\epsilon}{2}\right)^{-\delta} n \mathrm{E}\left|Z_{1, n}^{>}\right|^{\delta} \sum_{i=q_{n}+1}^{\infty} \mathrm{E}\left|C_{j}\right|^{\delta} .
$$

By Karamata's theorem $\lim _{n \rightarrow \infty} n \mathrm{E}\left|Z_{1, n}^{>}\right|^{\delta}=(1-\delta)^{-1}$ and hence from (3.1) we have $\lim _{n \rightarrow \infty} I_{2}=0$. Similarly we obtain $\lim _{n \rightarrow \infty} I_{4}=0$. This all allows us to conclude

$$
\lim _{n \rightarrow \infty} \mathrm{P}\left(\sup _{0 \leq t \leq 1}\left|V_{n, q_{n}}(t)-V_{n}(t)\right|>\epsilon\right)=0 .
$$

As before, Lemma 4.1 from Appendix and the modified proof of Theorem 2.1 (with $q$ replaced by $\left.q_{n}\right)$ imply $V_{n, q_{n}}(\cdot) \stackrel{d}{\rightarrow} \widetilde{C} V(\cdot)$ in $\left(D[0,1], d_{M_{2}}\right)$. Now the statement of the theorem follows by an application of Slutsky's theorem.

Remark 3.5. When the sequence of coefficients $\left(C_{j}\right)$ is deterministic, condition (3.4) is not needed. This was shown by Basrak and Krizmanić (2014), but their proof contains an error (i.e. they used Lemma 2 from Avram and Taqqu (1992), but the conditions needed to use this lemma were not fulfilled). Therefore in the proposition below we improve the proof of Theorem 3.1 in Basrak and Krizmanić (2014) in the case $\alpha \in[1,2)$, thus showing that condition (3.4) can be dropped if all coefficients of the moving average process are deterministic.

Proposition 3.6. Let $\left(Z_{i}\right)_{i \in \mathbb{Z}}$ be an i.i.d. sequence of regularly varying random variables with index $\alpha \in[1,2)$. Suppose that conditions (1.5) and (1.6) hold. Let $\left\{C_{i}, i=0,1,2, \ldots\right\}$ be a sequence of real numbers satisfying

$$
\sum_{j=0}^{\infty}\left|C_{j}\right|^{\delta}<\infty \quad \text { for some } \delta<\alpha, 0<\delta \leq 1,
$$

and

$$
0 \leq \sum_{i=0}^{s} C_{i} / \sum_{i=0}^{\infty} C_{i} \leq 1 \quad \text { for every } s=0,1,2 \ldots
$$

Then

$$
V_{n}(\cdot) \stackrel{d}{\rightarrow} C V(\cdot), \quad n \rightarrow \infty
$$

in $D[0,1]$ endowed with the $M_{2}$ topology, where $C=\sum_{j=0}^{\infty} C_{j}, V$ is an $\alpha$-stable Lévy process with characteristic triple $(0, \mu, b)$, with $\mu$ as in (1.9) and

$$
b=\left\{\begin{array}{cc}
0, & \alpha=1, \\
(p-r) \frac{\alpha}{1-\alpha}, & \alpha \in(1,2) .
\end{array}\right.
$$

Proof: Fix $q \in \mathbb{N}$, and define

$$
X_{i}^{q}=\sum_{j=0}^{q-1} C_{j} Z_{i-j}+C_{q}^{\prime} Z_{i-q}, \quad i \in \mathbb{Z},
$$

where $C_{q}^{\prime}=\sum_{j=q}^{\infty} C_{j}$, and

$$
V_{n, q}(t)=\sum_{i=1}^{\lfloor n t\rfloor} \frac{X_{i}^{q}}{a_{n}}, \quad t \in[0,1] .
$$


Since the coefficients $C_{0}, \ldots, C_{q-1}, C_{q}^{\prime}$ satisfy condition (2.1), an application of Theorem 2.1, adjusted to deterministic coefficients $C_{j}$, yields $V_{n, q}(\cdot) \stackrel{d}{\rightarrow} C V(\cdot)$ in $\left(D[0,1], d_{M_{2}}\right)$ as $n \rightarrow \infty$ (see also Theorem 2.1 in Basrak and Krizmanić, 2014). Therefore, in order to have $V_{n}(\cdot) \stackrel{d}{\rightarrow} C V(\cdot)$ in $D[0,1]$ with the $M_{2}$ topology, by a generalization of Slutsky's theorem we have to show that for every $\epsilon>0$

$$
\lim _{q \rightarrow \infty} \limsup _{n \rightarrow \infty} \mathrm{P}\left[d_{M_{2}}\left(V_{n, q}, V_{n}\right)>\epsilon\right]=0 .
$$

As before it suffices to show that

$$
\lim _{q \rightarrow \infty} \limsup _{n \rightarrow \infty} \mathrm{P}\left(\sup _{0 \leq t \leq 1}\left|V_{n, q}(t)-V_{n}(t)\right|>\epsilon\right)=0 .
$$

As in the proof of Theorem 3.1 we have

$$
\begin{gathered}
\mathrm{P}\left(\sup _{0 \leq t \leq 1}\left|V_{n, q}(t)-V_{n}(t)\right|>\epsilon\right) \\
\leq \mathrm{P}\left(\sup _{1 \leq k \leq n}\left|\sum_{i=1}^{k} \frac{C_{q}^{\prime \prime} Z_{i-q}}{a_{n}}\right|>\frac{\epsilon}{2}\right)+\mathrm{P}\left(\sup _{1 \leq k \leq n}\left|\sum_{i=1}^{k} \sum_{j=q+1}^{\infty} \frac{C_{j} Z_{i-j}}{a_{n}}\right|>\frac{\epsilon}{2}\right),
\end{gathered}
$$

where $C_{q}^{\prime \prime}=\sum_{i=q+1}^{\infty} C_{i}$. Take $\tau>0$ such that

$$
\left\{\begin{array}{l}
\alpha-\tau>\delta, \quad \text { if } \alpha=1, \\
\alpha-\tau>1, \quad \text { if } \alpha \in(1,2) .
\end{array}\right.
$$

Condition (3.18) implies $\sum_{j=0}^{\infty}\left|C_{j}\right|^{\alpha-\tau}<\infty$. Similarly $\sum_{j=0}^{\infty}\left|C_{j}\right|<\infty$. This implies that for large $q$ we have $\left|C_{q}^{\prime \prime}\right|<1$, which allows us to apply Lemma 2 in Avram and Taqqu (1992) to the first term on the right-hand side of (3.21), to obtain (for large $q$ )

$$
\begin{aligned}
\mathrm{P}\left(\sup _{1 \leq k \leq n}\left|\sum_{i=1}^{k} \frac{C_{q}^{\prime \prime} Z_{i-q}}{a_{n}}\right|>\frac{\epsilon}{2}\right) & \leq M\left(\frac{\epsilon}{2}\right)^{-(\alpha+\tau)} \frac{1}{n} \sum_{i=1}^{n}\left|C_{q}^{\prime \prime}\right|^{\alpha-\tau} \\
& =M\left(\frac{\epsilon}{2}\right)^{-(\alpha+\tau)}\left|C_{q}^{\prime \prime}\right|^{\alpha-\tau}
\end{aligned}
$$

where $M$ is a constant independent of $n$ and $q$. Using the following inequalities

$$
\begin{cases}\left|\sum_{i=1}^{m} a_{i}\right|^{s} \leq \sum_{i=1}^{m}\left|a_{i}\right|^{s}, & \text { if } s \leq 1, \\ \left|\sum_{i=1}^{m} a_{i}\right|^{s} \leq \sum_{i=1}^{m}\left|a_{i}\right|, & \text { if } s>1 \text { and }\left|\sum_{i=1}^{m} a_{i}\right|<1,\end{cases}
$$

we have

$$
\left|C_{q}^{\prime \prime}\right|^{\alpha-\tau} \leq\left\{\begin{array}{lc}
\sum_{j=q+1}^{\infty}\left|C_{j}\right|^{\alpha-\tau}, & \text { if } \alpha=1, \\
\sum_{j=q+1}^{\infty}\left|C_{j}\right|, & \text { if } \alpha \in(1,2),
\end{array}\right.
$$

yielding $\lim _{q \rightarrow \infty}\left|C_{q}^{\prime \prime}\right|^{\alpha-\tau}=0$. Now from (3.22) we obtain

$$
\lim _{q \rightarrow \infty} \limsup _{n \rightarrow \infty} \mathrm{P}\left(\sup _{1 \leq k \leq n}\left|\sum_{i=1}^{k} \frac{C_{q}^{\prime \prime} Z_{i-q}}{a_{n}}\right|>\frac{\epsilon}{2}\right)=0 .
$$


Note that the second term on the right-hand side of (3.21) is bounded above by

$$
\mathrm{P}\left(\sup _{1 \leq k \leq n}\left|\sum_{i=1}^{k} \sum_{j=q+1}^{\infty} \frac{C_{j}^{+} Z_{i-j}}{a_{n}}\right|>\frac{\epsilon}{4}\right)+\mathrm{P}\left(\sup _{1 \leq k \leq n}\left|\sum_{i=1}^{k} \sum_{j=q+1}^{\infty} \frac{C_{j}^{-} Z_{i-j}}{a_{n}}\right|>\frac{\epsilon}{4}\right),
$$

where $C_{j}^{+}=C_{j} \vee 0$ and $C_{j}^{-}=\left(-C_{j}\right) \vee 0$. In the sequel we consider only the first of these two probabilities since the other one can be handled in the same manner.

Assume first $\alpha \in(1,2)$. Recall $\widetilde{Z}_{i, n}^{\leq}$and $\widetilde{Z}_{i, n}^{>}$from the proof of Lemma 3.3, and note

$$
\begin{aligned}
& \mathrm{P}\left(\sup _{1 \leq k \leq n}\left|\sum_{i=1}^{k} \sum_{j=q+1}^{\infty} \frac{C_{j}^{+} Z_{i-j}}{a_{n}}\right|>\frac{\epsilon}{4}\right) \\
& \leq \mathrm{P}\left(\sup _{1 \leq k \leq n}\left|\sum_{i=1}^{k} \sum_{j=q+1}^{\infty} C_{j}^{+} \widetilde{Z}_{i-j, n}^{\leq}\right|>\frac{\epsilon}{8}\right)+\mathrm{P}\left(\sup _{1 \leq k \leq n}\left|\sum_{i=1}^{k} \sum_{j=q+1}^{\infty} C_{j}^{+} \widetilde{Z}_{i-j, n}^{>}\right|>\frac{\epsilon}{8}\right)
\end{aligned}
$$

Since the coefficients $C_{j}^{+}$are nonnegative, the moving average processes

$$
Y_{i, n, q}^{\leq}:=\sum_{j=q+1}^{\infty} C_{j}^{+} \widetilde{Z}_{i-j, n}^{\leq}, \quad i=1,2, \ldots
$$

are associated, as nondecreasing functions of independent random variables (see Esary et al., 1967). Thus the sequence $\left(\sum_{i=1}^{k} Y_{i, n, q}^{\leq}\right)_{k}$ is a demimartingale (see Section 2.1 in Prakasa Rao, 2012), and hence by Markov's inequality and the maximal inequality for demimartingales

$$
\mathrm{E}\left(\sup _{1 \leq k \leq n}\left|S_{k}\right|\right)^{\kappa} \leq\left(\frac{\kappa}{\kappa-1}\right)^{\kappa} \mathrm{E}\left|S_{n}\right|^{\kappa}
$$

which holds for $\kappa>1$ and $\left(S_{k}\right)_{k}$ a demimartingale (see for example Corollary 2.4 in Wang et al., 2010) we obtain

$$
\mathrm{P}\left(\sup _{1 \leq k \leq n}\left|\sum_{i=1}^{k} \sum_{j=q+1}^{\infty} C_{j}^{+} \widetilde{Z}_{i-j, n}^{\leq}\right|>\frac{\epsilon}{8}\right) \leq\left(\frac{\epsilon}{8}\right)^{-(\alpha+\tau)}\left(\frac{\alpha+\tau}{\alpha+\tau-1}\right)^{\alpha+\tau} \mathrm{E}\left|\sum_{i=1}^{n} Y_{i, n, q}^{\leq}\right|^{\alpha+\tau}
$$

and similarly

$$
\mathrm{P}\left(\sup _{1 \leq k \leq n}\left|\sum_{i=1}^{k} \sum_{j=q+1}^{\infty} C_{j}^{+} \widetilde{Z}_{i-j, n}^{>}\right|>\frac{\epsilon}{8}\right) \leq\left(\frac{\epsilon}{8}\right)^{-(\alpha-\tau)}\left(\frac{\alpha-\tau}{\alpha-\tau-1}\right)^{\alpha-\tau} \mathrm{E}\left|\sum_{i=1}^{n} Y_{i, n, q}^{>}\right|^{\alpha-\tau}
$$

where $Y_{i, n, q}^{>}:=\sum_{j=q+1}^{\infty} C_{j}^{+} \widetilde{Z}_{i-j, n}^{>}$. By standard changes of variables and order of summation we have

$$
\sum_{i=1}^{n} Y_{i, n, q}^{\leq}=\sum_{i=-\infty}^{n-1}\left(\sum_{j=q+1+(-i) \vee 0}^{q+n-i} C_{j}^{+}\right) \widetilde{Z}_{i-q, n}^{\leq}
$$


Note that $\left(\left(\sum_{j=q+1+(-i) \vee 0}^{q+n-i} C_{j}^{+}\right) \widetilde{Z}_{i-q, n}^{\leq}\right)_{i}$ is a martingale difference sequence, and thus by the Bahr-Esseen inequality we obtain

$$
\mathrm{E}\left|\sum_{i=1}^{n} Y_{i, n, q}^{\leq}\right|^{\alpha+\tau} \leq 2 \sum_{i=-\infty}^{n-1}\left(\sum_{j=q+1+(-i) \vee 0}^{q+n-i} C_{j}^{+}\right)^{\alpha+\tau} \mathrm{E}\left|\widetilde{Z}_{i-q, n}^{\leq}\right|^{\alpha+\tau}
$$

Noting that for large $q, \sum_{j=q+1+(-i) \vee 0}^{q+n-i} C_{j}^{+}<1$, the second inequality in (3.23) yields that (for large $q$ )

$$
\mathrm{E}\left|\sum_{i=1}^{n} Y_{i, n, q}^{\leq}\right|^{\alpha+\tau} \leq 2\left|\widetilde{Z}_{1, n}^{\leq}\right|^{\alpha+\tau} \sum_{i=-\infty}^{n-1} \sum_{j=q+1+(-i) \vee 0}^{q+n-i} C_{j}^{+} .
$$

Now note that every $C_{j}^{+}$, for $j \geq q+1$, appears in the sum $\sum_{i=-\infty}^{n-1} \sum_{j=q+1+(-i) \vee 0}^{q+n-i} C_{j}^{+}$ at most $n$ times, and therefore

$$
\mathrm{E}\left|\sum_{i=1}^{n} Y_{i, n, q}^{\leq}\right|^{\alpha+\tau} \leq 2 n\left|\widetilde{Z}_{1, n}^{\leq}\right|^{\alpha+\tau} \sum_{j=q+1}^{\infty} C_{j}^{+}
$$

Similarly we obtain

$$
\mathrm{E}\left|\sum_{i=1}^{n} Y_{i, n, q}^{>}\right|^{\alpha-\tau} \leq 2 n\left|\widetilde{Z}_{1, n}^{>}\right|^{\alpha-\tau} \sum_{j=q+1}^{\infty} C_{j}^{+}
$$

Jensen's inequality, as in (3.9), yields

$$
\mathrm{E}\left|\widetilde{Z}_{1, n}^{\leq}\right|^{\alpha+\tau} \leq 2^{\alpha+\tau+1} \mathrm{E}\left|Z_{1, n}^{\leq}\right|^{\alpha+\tau}
$$

and similarly

$$
\mathrm{E}\left|\widetilde{Z}_{1, n}^{>}\right|^{\alpha-\tau} \leq 2^{\alpha-\tau+1} \mathrm{E}\left|Z_{1, n}^{>}\right|^{\alpha-\tau}
$$

Collecting all these facts, from (3.26) and (3.27) we obtain, for large $q$,

$$
\begin{aligned}
& \mathrm{P}\left(\sup _{1 \leq k \leq n}\left|\sum_{i=1}^{k} \sum_{j=q+1}^{\infty} C_{j}^{+} \widetilde{Z}_{i-j, n}^{\leq}\right|>\frac{\epsilon}{8}\right) \\
& \quad \leq 2^{\alpha+\tau+2}\left(\frac{\epsilon}{8}\right)^{-(\alpha+\tau)}\left(\frac{\alpha+\tau}{\alpha+\tau-1}\right)^{\alpha+\tau} n\left|Z_{1, n}^{\leq}\right|^{\alpha+\tau} \sum_{j=q+1}^{\infty} C_{j}^{+}
\end{aligned}
$$

and

$$
\begin{aligned}
& \mathrm{P}\left(\sup _{1 \leq k \leq n}\left|\sum_{i=1}^{k} \sum_{j=q+1}^{\infty} C_{j}^{+} \widetilde{Z}_{i-j, n}^{>}\right|>\frac{\epsilon}{8}\right) \\
& \quad \leq 2^{\alpha-\tau+2}\left(\frac{\epsilon}{8}\right)^{-(\alpha-\tau)}\left(\frac{\alpha-\tau}{\alpha-\tau-1}\right)^{\alpha-\tau} n\left|Z_{1, n}^{>}\right|^{\alpha-\tau} \sum_{j=q+1}^{\infty} C_{j}^{+}
\end{aligned}
$$

From (3.25), (3.28) and (3.29) we see that for some positive constant $M^{\prime}$ the following inequality holds for large $q$

$$
\mathrm{P}\left(\sup _{1 \leq k \leq n}\left|\sum_{i=1}^{k} \sum_{j=q+1}^{\infty} \frac{C_{j}^{+} Z_{i-j}}{a_{n}}\right|>\frac{\epsilon}{4}\right) \leq M^{\prime}\left(n\left|Z_{1, n}^{\leq}\right|^{\alpha+\tau}+n\left|Z_{1, n}^{>}\right|^{\alpha-\tau}\right) \sum_{j=q+1}^{\infty} C_{j}^{+} .
$$


By Karamata's theorem $n \mathrm{E}\left|Z_{1, n}^{\leq}\right|^{\alpha+\tau} \rightarrow \alpha / \tau$ and $n \mathrm{E}\left|Z_{1, n}^{>}\right|^{\alpha-\tau} \rightarrow \alpha / \tau$, as $n \rightarrow \infty$. Therefore, since $\sum_{j=q+1}^{\infty} C_{j}^{+} \leq \sum_{j=q+1}^{\infty}\left|C_{j}\right| \rightarrow 0$ as $q \rightarrow \infty$, we have

$$
\lim _{q \rightarrow \infty} \limsup _{n \rightarrow \infty} \mathrm{P}\left(\sup _{1 \leq k \leq n}\left|\sum_{i=1}^{k} \sum_{j=q+1}^{\infty} \frac{C_{j}^{+} Z_{i-j}}{a_{n}}\right|>\frac{\epsilon}{4}\right)=0 .
$$

Hence we conclude

$$
\lim _{q \rightarrow \infty} \limsup _{n \rightarrow \infty} \mathrm{P}\left(\sup _{1 \leq k \leq n}\left|\sum_{i=1}^{k} \sum_{j=q+1}^{\infty} \frac{C_{j} Z_{i-j}}{a_{n}}\right|>\frac{\epsilon}{2}\right)=0 .
$$

Now, from $(3.21),(3.24)$ and $(3.30)$ follows $(3.20)$, which means that $V_{n}(\cdot) \stackrel{d}{\rightarrow}$ $C V(\cdot)$ in $D[0,1]$ with the $M_{2}$ topology.

Assume now $\alpha=1$. Relation (3.26) holds also in this case, but for (3.27) we need a different argument since $\alpha-\tau<1$, and thus we can not use the maximal inequality for demimartingales. By Markov's inequality and the first inequality in (3.23) we have

$$
\begin{aligned}
\mathrm{P}\left(\sup _{1 \leq k \leq n}\left|\sum_{i=1}^{k} \sum_{j=q+1}^{\infty} C_{j}^{+} \widetilde{Z}_{i-j, n}^{>}\right|>\frac{\epsilon}{8}\right) & \leq \mathrm{P}\left(\sum_{i=1}^{n} \sum_{j=q+1}^{\infty} C_{j}^{+}\left|\widetilde{Z}_{i-j, n}^{>}\right|>\frac{\epsilon}{8}\right) \\
& \leq\left(\frac{\epsilon}{8}\right)^{-(\alpha-\tau)} \mathrm{E}\left(\sum_{i=1}^{n} \sum_{j=q+1}^{\infty} C_{j}^{+}\left|\widetilde{Z}_{i-j, n}^{>}\right|\right)^{\alpha-\tau}, \\
& \leq\left(\frac{\epsilon}{8}\right)^{-(\alpha-\tau)} \mathrm{E}\left|\widetilde{Z}_{1, n}^{>}\right|^{\alpha-\tau} \sum_{i=1}^{n} \sum_{j=q+1}^{\infty}\left(C_{j}^{+}\right)^{\alpha-\tau} \\
& \leq\left(\frac{\epsilon}{8}\right)^{-(\alpha-\tau)} n \mathrm{E}\left|\widetilde{Z}_{1, n}^{>}\right|^{\alpha-\tau} \sum_{j=q+1}^{\infty}\left|C_{j}\right|^{\alpha-\tau} .
\end{aligned}
$$

From the symmetry of $Z_{1}$, Karamata's theorem and (1.7) we obtain, as $n \rightarrow \infty$,

$$
n \mathrm{E}\left|\widetilde{Z}_{1, n}^{>}\right|^{\alpha-\tau}=n \mathrm{E}\left|Z_{1, n}^{>}\right|^{\alpha-\tau}=\frac{\mathrm{E}\left(\left|Z_{1}\right|^{\alpha-\tau} 1_{\left\{\left|Z_{1}\right|>a_{n}\right\}}\right)}{a_{n}^{\alpha-\tau} \mathrm{P}\left(\left|Z_{1}\right|>a_{n}\right)} \cdot n \mathrm{P}\left(\left|Z_{1}\right|>a_{n}\right) \rightarrow \frac{\alpha}{\tau} .
$$

Therefore, since $\lim _{q \rightarrow \infty} \sum_{j=q+1}^{\infty}\left|C_{j}\right|^{\alpha-\tau}=0$, we have

$$
\lim _{q \rightarrow \infty} \limsup _{n \rightarrow \infty} \mathrm{P}\left(\sup _{1 \leq k \leq n}\left|\sum_{i=1}^{k} \sum_{j=q+1}^{\infty} C_{j}^{+} \widetilde{Z}_{i-j, n}^{>}\right|>\frac{\epsilon}{8}\right)=0,
$$

and as in the case $\alpha \in(1,2)$ it follows that $V_{n}(\cdot) \stackrel{d}{\rightarrow} C V(\cdot)$ in $D[0,1]$ with the $M_{2}$ topology. This completes the proof.

\section{Appendix}

We provide a technical result used in the proof of Theorem 3.1 and an example of moving average process with heavy-tailed innovations and deterministic coefficients for which the $M_{1}$ convergence of the corresponding partial sum process does not hold (but for which the $M_{2}$ convergence holds). 
Lemma 4.1. Let $Z_{1}$ be a regularly varying random variable with index $\alpha \in[1,2)$ and $\left(a_{n}\right)$ a sequence of positive real numbers such that (1.7) holds. Let $q_{n}=$ $\left\lfloor n^{1 / 10}\right\rfloor, n \in \mathbb{N}$. Then

$$
\lim _{n \rightarrow \infty} n q_{n}^{2}\left[\mathrm{P}\left(\left|Z_{1}\right|>\frac{a_{n}}{q_{n}}\right)\right]^{2}=0 .
$$

Proof: By (1.1) and (1.7) we have

$$
\lim _{n \rightarrow \infty} n a_{n}^{-\alpha} L\left(a_{n}\right)=1
$$

Since $L$ is a slowly varying function, it holds that for all $s>0$ and $t \in \mathbb{R}$, as $x \rightarrow \infty, x^{s}[L(x)]^{t} \rightarrow \infty$ and $x^{-s}[L(x)]^{t} \rightarrow 0$ (Bingham et al., 1989, Proposition 1.3.6). Hence $a_{n}^{2-\alpha} L\left(a_{n}\right) \rightarrow \infty$ as $n \rightarrow \infty$, and since by (4.1)

$$
\lim _{n \rightarrow \infty} \frac{n}{a_{n}^{2}} a_{n}^{2-\alpha} L\left(a_{n}\right)=1,
$$

it follows that $n / a_{n}^{2} \rightarrow 0$ as $n \rightarrow \infty$. This yields

$$
\frac{a_{n}}{q_{n}}=\sqrt{\frac{a_{n}^{2}}{n}} \cdot \frac{\sqrt{n}}{q_{n}} \rightarrow \infty \quad \text { as } n \rightarrow \infty,
$$

since by the definition of the sequence $\left(q_{n}\right), \sqrt{n} / q_{n} \rightarrow \infty$. Thus for $u>0, M_{n}(u):=$ $\left(a_{n} / q_{n}\right)^{-u}\left[L\left(a_{n} / q_{n}\right)\right]^{2} \rightarrow 0$ as $n \rightarrow \infty$.

From (1.1) we obtain

$$
n q_{n}^{2}\left[\mathrm{P}\left(\left|Z_{1}\right|>\frac{a_{n}}{q_{n}}\right)\right]^{2}=n q_{n}^{2}\left(\frac{a_{n}}{q_{n}}\right)^{-2 \alpha}\left[L\left(\frac{a_{n}}{q_{n}}\right)\right]^{2}=n q_{n}^{2}\left(\frac{a_{n}}{q_{n}}\right)^{-2 \alpha+u} M_{n}(u) .
$$

By (4.1) we have

$$
a_{n}^{\alpha} \geq K n L\left(a_{n}\right)
$$

for some positive constant $K$ independent of $n$, and hence taking some $v>0$ such that $u+v<2 \alpha$ we obtain

$$
\begin{aligned}
& n q_{n}^{2}\left[\mathrm{P}\left(\left|Z_{1}\right|>\frac{a_{n}}{q_{n}}\right)\right]^{2} \\
& =n q_{n}^{2+2 \alpha-u}\left(a_{n}^{\alpha}\right)^{-2+(u+v) / \alpha} a_{n}^{-v} M_{n}(u) \\
& \leq K^{-2+(u+v) \alpha} q_{n}^{2+2 \alpha-u} n^{-1+(u+v) / \alpha} a_{n}^{-v}\left[L\left(a_{n}\right)\right]^{-2+(u+v) / \alpha} M_{n}(u) \\
& =q_{n}^{2+2 \alpha-u} n^{-1+(u+v) / \alpha} M_{n}(u, v) \\
& \leq n^{(2+2 \alpha-u) / 10-1+(u+v) / \alpha} M_{n}(u, v) .
\end{aligned}
$$

where

$$
M_{n}(u, v):=K^{-2+(u+v) \alpha} a_{n}^{-v}\left[L\left(a_{n}\right)\right]^{-2+(u+v) / \alpha} M_{n}(u) \rightarrow 0, \quad \text { as } n \rightarrow \infty .
$$

Now let $u=1 / 5$ and $v=1 / 5$, and note that for this choice of $u$ and $v$ it holds that

$$
\frac{2+2 \alpha-u}{10}-1+\frac{u+v}{\alpha} \leq-\frac{1}{50}<0 .
$$

Therefore from (4.2) we obtain

$$
\lim _{n \rightarrow \infty} n q_{n}^{2}\left[\mathrm{P}\left(\left|Z_{1}\right|>\frac{a_{n}}{q_{n}}\right)\right]^{2}=0 .
$$


Example 4.2. Let $\left(Z_{i}\right)_{i}$ be a sequence of i.i.d. regularly varying random variables with index of regular variation $\alpha \in(0,2)$. Define the moving average process

$$
X_{i}=Z_{i}-Z_{i-1}+Z_{i-2}, \quad i \in \mathbb{Z} .
$$

We will show that $V_{n}$, as defined in (2.2), does not converge in distribution under the $M_{1}$ topology on $D[0,1]$. For this, according to Skorohod (1956) (see also Proposition 2 in Avram and Taqqu, 1992), it suffices to show that

$$
\lim _{\delta \rightarrow 0} \limsup _{n \rightarrow \infty} \mathrm{P}\left(\omega_{\delta}\left(V_{n}\right)>\epsilon\right)>0
$$

for some $\epsilon>0$, where

$$
\omega_{\delta}(x)=\sup _{\substack{t_{1} \leq t \leq t_{2} \\ 0 \leq t_{2}-t_{1} \leq \delta}} M\left(x\left(t_{1}\right), x(t), x\left(t_{2}\right)\right)
$$

$(x \in D[0,1], \delta>0)$ and

$$
M\left(x_{1}, x_{2}, x_{3}\right)= \begin{cases}0, & \text { if } x_{2} \in\left[x_{1}, x_{3}\right], \\ \min \left\{\left|x_{2}-x_{1}\right|,\left|x_{3}-x_{2}\right|\right\}, & \text { otherwise }\end{cases}
$$

Note that $M\left(x_{1}, x_{2}, x_{3}\right)$ is the distance from $x_{2}$ to $\left[x_{1}, x_{3}\right]$, and $\omega_{\delta}(x)$ is the $M_{1}$ oscillation of $x$. To show (4.3) we use, with appropriate modifications, the procedure of Avram and Taqqu (1992) in the proof of their Theorem 1.

Let $i^{\prime}=i^{\prime}(n)$ be the index at which $\max _{1 \leq i \leq n-1}\left|Z_{i}\right|$ is obtained. Fix $\epsilon>0$ and introduce the events

$$
A_{n, \epsilon}=\left\{\left|Z_{i^{\prime}}\right|>\epsilon a_{n}\right\}=\left\{\max _{1 \leq i \leq n-1}\left|Z_{i}\right|>\epsilon a_{n}\right\}
$$

and

$$
B_{n, \epsilon}=\left\{\left|Z_{i^{\prime}}\right|>\epsilon a_{n} \text { and } \exists l \neq 0,-i^{\prime}-1 \leq l \leq 1 \text {, such that }\left|Z_{i^{\prime}+l}\right|>\epsilon a_{n} / 4\right\} .
$$

Using the facts that $\left(Z_{i}\right)$ is an i.i.d. sequence and $n \mathrm{P}\left(Z_{1}>\lambda a_{n}\right) \rightarrow \lambda^{-\alpha}$ as $n \rightarrow \infty$ for $\lambda>0$ (which follows from the regular variation property of $Z_{1}$ and (1.7)) we get

$$
\lim _{n \rightarrow \infty} \mathrm{P}\left(A_{n, \epsilon}\right)=1-e^{-\epsilon^{-\alpha}}
$$

and

$$
\limsup _{n \rightarrow \infty} \mathrm{P}\left(B_{n, \epsilon}\right) \leq \frac{\epsilon^{-2 \alpha}}{4^{-\alpha}}
$$

(see Example 5.1 in Krizmanić, 2014).

On the event $A_{n, \epsilon} \backslash B_{n, \epsilon}$ one has $\left|Z_{i^{\prime}}\right|>\epsilon a_{n}$ and $\left|Z_{i^{\prime}+l}\right| \leq \epsilon a_{n} / 4$ for every $l \neq 0$, $-i^{\prime}-1 \leq l \leq 1$, and hence

$$
\left|V_{n}\left(\frac{i^{\prime}}{n}\right)-V_{n}\left(\frac{i^{\prime}-1}{n}\right)\right|=\frac{\left|X_{i^{\prime}}\right|}{a_{n}}=\frac{\left|Z_{i^{\prime}}-Z_{i^{\prime}-1}+Z_{i^{\prime}-2}\right|}{a_{n}}>\frac{\epsilon}{2}
$$

and

$$
\left|V_{n}\left(\frac{i^{\prime}+1}{n}\right)-V_{n}\left(\frac{i^{\prime}}{n}\right)\right|=\frac{\left|X_{i^{\prime}+1}\right|}{a_{n}}=\frac{\left|Z_{i^{\prime}+1}-Z_{i^{\prime}}+Z_{i^{\prime}-1}\right|}{a_{n}}>\frac{\epsilon}{2} .
$$

Further, we claim that on the event $A_{n, \epsilon} \backslash B_{n, \epsilon}$ it also holds that

$$
V_{n}\left(\frac{i^{\prime}}{n}\right) \notin\left[V_{n}\left(\frac{i^{\prime}-1}{n}\right), V_{n}\left(\frac{i^{\prime}+1}{n}\right)\right] .
$$


If this is not the case, then

$$
V_{n}\left(\frac{i^{\prime}-1}{n}\right) \leq V_{n}\left(\frac{i^{\prime}}{n}\right) \leq V_{n}\left(\frac{i^{\prime}+1}{n}\right),
$$

i.e.

$$
0 \leq \frac{X_{i^{\prime}}}{a_{n}} \leq \frac{X_{i^{\prime}}+X_{i^{\prime}+1}}{a_{n}}
$$

Therefore

$$
\frac{X_{i^{\prime}}}{a_{n}}=\frac{Z_{i^{\prime}}-Z_{i^{\prime}-1}+Z_{i^{\prime}-2}}{a_{n}} \geq 0 \quad \text { and } \quad \frac{X_{i^{\prime}+1}}{a_{n}}=\frac{Z_{i^{\prime}+1}-Z_{i^{\prime}}+Z_{i^{\prime}-1}}{a_{n}} \geq 0,
$$

and this implies

$$
\frac{Z_{i^{\prime}}}{a_{n}}>\frac{\epsilon}{2} \quad \text { and } \quad \frac{Z_{i^{\prime}}}{a_{n}}<-\frac{\epsilon}{2}
$$

which is not possible. Thus relation (4.8) holds, and it implies

$$
\begin{aligned}
M\left(V_{n}\left(\frac{i^{\prime}-1}{n}\right),\right. & \left.V_{n}\left(\frac{i^{\prime}}{n}\right), V_{n}\left(\frac{i^{\prime}+1}{n}\right)\right) \\
& =\min \left\{\left|V_{n}\left(\frac{i^{\prime}}{n}\right)-V_{n}\left(\frac{i^{\prime}-1}{n}\right)\right|,\left|V_{n}\left(\frac{i^{\prime}+1}{n}\right)-V_{n}\left(\frac{i^{\prime}}{n}\right)\right|\right\} .
\end{aligned}
$$

Taking into account (4.6) and (4.7) we obtain

$$
\begin{aligned}
\omega_{2 / n}\left(V_{n}\right)= & \sup _{\substack{t_{1} \leq t \leq t_{2} \\
0 \leq t_{2}-t_{1} \leq 2 / n}} M\left(V_{n}\left(t_{1}\right), V_{n}(t), V_{n}\left(t_{2}\right)\right) \\
\geq & M\left(V_{n}\left(\frac{i^{\prime}-1}{n}\right), V_{n}\left(\frac{i^{\prime}}{n}\right), V_{n}\left(\frac{i^{\prime}+1}{n}\right)\right)>\frac{\epsilon}{2}
\end{aligned}
$$

on the event $A_{n, \epsilon} \backslash B_{n, \epsilon}$. Therefore, since $\omega_{\delta}$ is nondecreasing in $\delta$, it holds that

$$
\begin{aligned}
\liminf _{n \rightarrow \infty} \mathrm{P}\left(A_{n, \epsilon} \backslash B_{n, \epsilon}\right) & \leq \liminf _{n \rightarrow \infty} \mathrm{P}\left(\omega_{2 / n}\left(V_{n}\right)>\epsilon / 2\right) \\
& \leq \lim _{\delta \rightarrow 0} \limsup _{n \rightarrow \infty} \mathrm{P}\left(\omega_{\delta}\left(V_{n}\right)>\epsilon / 2\right) .
\end{aligned}
$$

Since $x^{2 \alpha}\left(1-e^{-x^{-\alpha}}\right)$ tends to infinity as $x \rightarrow \infty$, we can find $\epsilon>0$ such that $\epsilon^{2 \alpha}\left(1-e^{-\epsilon^{-\alpha}}\right)>4^{\alpha}$, i.e.

$$
1-e^{-\epsilon^{-\alpha}}>\frac{\epsilon^{-2 \alpha}}{4^{-\alpha}}
$$

For this $\epsilon$, by relations (4.4) and (4.5), it holds that

$$
\lim _{n \rightarrow \infty} \mathrm{P}\left(A_{n, \epsilon}\right)>\limsup _{n \rightarrow \infty} \mathrm{P}\left(B_{n, \epsilon}\right),
$$

i.e.

$$
\liminf _{n \rightarrow \infty} \mathrm{P}\left(A_{n, \epsilon} \backslash B_{n, \epsilon}\right) \geq \lim _{n \rightarrow \infty} \mathrm{P}\left(A_{n, \epsilon}\right)-\limsup _{n \rightarrow \infty} \mathrm{P}\left(B_{n, \epsilon}\right)>0 .
$$

Therefore by (4.9) we obtain

$$
\lim _{\delta \rightarrow 0} \limsup _{n \rightarrow \infty} \mathrm{P}\left(\omega_{\delta}\left(V_{n}\right)>\epsilon / 2\right)>0
$$

and relation (4.3) holds, which means that $V_{n}$ does not converge in distribution in $D[0,1]$ endowed with the $M_{1}$ topology. 


\section{Acknowledgements}

The author would like to thank the anonymous referee for the careful reading of the manuscript and helpful remarks which improved the paper.

\section{References}

F. Avram and M. S. Taqqu. Probability bounds for $M$-Skorohod oscillations. Stochastic Process. Appl. 33 (1), 63-72 (1989). MR1027108.

F. Avram and M. S. Taqqu. Weak convergence of sums of moving averages in the $\alpha$-stable domain of attraction. Ann. Probab. 20 (1), 483-503 (1992). MR1143432.

B. Basrak and D. Krizmanić. A limit theorem for moving averages in the $\alpha$ stable domain of attraction. Stochastic Process. Appl. 124 (2), 1070-1083 (2014). MR3138607.

B. Basrak, D. Krizmanić and J. Segers. A functional limit theorem for dependent sequences with infinite variance stable limits. Ann. Probab. 40 (5), 2008-2033 (2012). MR3025708.

P. Billingsley. Convergence of probability measures. John Wiley \& Sons, Inc., New York-London-Sydney (1968). MR0233396.

N. H. Bingham, C. M. Goldie and J. L. Teugels. Regular variation, volume 27 of Encyclopedia of Mathematics and its Applications. Cambridge University Press, Cambridge (1989). ISBN 0-521-37943-1. MR1015093.

S. D. Chatterji. An $L^{p}$-convergence theorem. Ann. Math. Statist. 40, 1068-1070 (1969). MR0251769.

R. Durrett. Probability: theory and examples. Duxbury Press, Belmont, CA, second edition (1996). ISBN 0-534-24318-5. MR1609153.

J. D. Esary, F. Proschan and D. W. Walkup. Association of random variables, with applications. Ann. Math. Statist. 38, 1466-1474 (1967). MR0217826.

H. Hult and G. Samorodnitsky. Tail probabilities for infinite series of regularly varying random vectors. Bernoulli 14 (3), 838-864 (2008). MR2537814.

O. Kallenberg. Foundations of modern probability. Probability and its Applications (New York). Springer-Verlag, New York (1997). ISBN 0-387-94957-7. MR1464694.

D. Krizmanić. Weak convergence of partial maxima processes in the $M_{1}$ topology. Extremes 17 (3), 447-465 (2014). MR3252821.

R. Kulik. Limit theorems for moving averages with random coefficients and heavytailed noise. J. Appl. Probab. 43 (1), 245-256 (2006). MR2225064.

B. L. S. Prakasa Rao. Associated sequences, demimartingales and nonparametric inference. Probability and its Applications. Birkhäuser/Springer, Basel (2012). ISBN 978-3-0348-0239-0; 978-3-0348-0240-6. MR3025761.

S. I. Resnick. Heavy-tail phenomena. Springer Series in Operations Research and Financial Engineering. Springer, New York (2007). ISBN 978-0-387-24272-9; 0387-24272-4. MR2271424.

A. V. Skorohod. Limit theorems for stochastic processes. Teor. Veroyatnost. $i$ Primenen. 1, 289-319 (1956). MR0084897.

X. Wang, S. Hu, T. Zhao and W. Yang. Doob's type inequality and strong law of large numbers for demimartingales. J. Inequal. Appl. pages Art. ID 838301, 11 (2010). MR2600190. 
W. Whitt. Stochastic-process limits. An introduction to stochastic-process limits and their application to queues. Springer Series in Operations Research. SpringerVerlag, New York (2002). ISBN 0-387-95358-2. MR1876437. 